\title{
On the nature of outflows in intermediate-mass protostars: a case study of IRAS $20050+2720$ *
}

\author{
M. T. Beltrán ${ }^{1}$, R. Estalella ${ }^{1}$, J. M. Girart ${ }^{2}$, P. T. P. $\mathrm{Ho}^{3,4}$, and G. Anglada ${ }^{5}$
}

\author{
1 Departament d'Astronomia i Meteorologia, Universitat de Barcelona, Martí i Franquès 1, 08028 Barcelona, Catalunya, Spain \\ e-mail: mbeltran@am.ub.es \\ 2 Institut de Ciències de l'Espai (CSIC-IEEC), Campus UAB, Facultat de Ciències, Torre C-5, 08193, Bellaterra, Catalunya, Spain \\ 3 Harvard-Smithsonian Center for Astrophysics, 60 Garden Street, Cambridge, MA 02138, USA \\ 4 Academia Sinica, Institute of Astronomy and Astrophysics, PO Box 23-141, Taipei 106, Taiwan, ROC \\ 5 Instituto de Astrofísica de Andalucía, CSIC, Camino Bajo de Huétor 50, 18008 Granada, Spain
}

Received 8 June 2007 / Accepted 11 December 2007

\begin{abstract}
Context. This is the third in a series of papers devoted to studying intermediate-mass molecular outflows and their powering sources in detail and with high-angular resolution.

Aims. This paper studies the intermediate-mass YSO IRAS 20050+2720 and its molecular outflow and puts the results of this and the previous studied sources in the context of intermediate-mass star formation.

Methods. We carried out VLA observations of the $7 \mathrm{~mm}$ continuum emission and OVRO observations of the $2.7 \mathrm{~mm}$ continuum emission, $\mathrm{CO}(J=1 \rightarrow 0), \mathrm{C}^{18} \mathrm{O}(J=1 \rightarrow 0)$, and $\mathrm{HC}_{3} \mathrm{~N}(J=12 \rightarrow 11)$ to map the core towards IRAS 20050+2720. The highangular resolution of the observations allowed us to derive the properties of the dust emission, the molecular outflow, and the dense protostellar envelope. By adding this source to the sample of intermediate-mass protostars with outflows, we compared their properties and evolution with those of lower mass counterparts.

Results. The $2.7 \mathrm{~mm}$ continuum emission has been resolved into three sources, labeled OVRO 1, OVRO 2, and OVRO 3 . Two of them, OVRO 1 and OVRO 2, have also been detected at $7 \mathrm{~mm}$. OVRO 3, which is located close to the $\mathrm{C}^{18} \mathrm{O}$ emission peak, could be associated with IRAS $20050+2720$. The mass of the sources, estimated from the dust continuum emission, is $6.5 M_{\odot}$ for OVRO 1 , $1.8 M_{\odot}$ for OVRO 2, and $1.3 M_{\odot}$ for OVRO 3. The CO $(J=1 \rightarrow 0)$ emission traces two bipolar outflows within the OVRO field of view, a roughly east-west bipolar outflow, labeled A, driven by the intermediate-mass source OVRO 1, and a northeast-southwest bipolar outflow, labeled B, probably powered by a YSO engulfed in the circumstellar envelope surrounding OVRO 1.

Conclusions. The multiplicity of sources observed towards IRAS $20050+2720$ appears to be typical of intermediate-mass protostars, which form in dense clustered environments. In some cases, as for example IRAS 20050+2720, intermediate-mass protostars would start forming after a first generation of low-mass stars has completed their main accretion phase. The properties of intermediate-mass protostars and their outflows are not significantly different from those of low-mass stars. Although intermediate-mass outflows are intrinsically more energetic than those driven by low-mass YSOs, they do not show intrinsically more complex morphologies when observed at high angular resolution. Known intermediate-mass protostars do not form a homogeneous group. Some objects are likely in an earlier evolutionary stage as suggested by the infrared emission and the outflow properties.
\end{abstract}

Key words. ISM: individual objects: IRAS 20050+2720 - ISM: jets and outflows - stars: circumstellar matter - stars: formation

\section{Introduction}

Molecular outflow is a ubiquitous phenomenon during the earliest stages of the formation of stars of all masses and luminosities. During the last few decades, many efforts have been devoted to studying and describing the physical properties of embedded low-mass protostars and their molecular outflows (e.g., Bachiller 1996; Richer et al. 2000). In recent years, high-mass molecular outflows have also been studied in detail, and many surveys have been carried out towards massive star-forming regions to achieve a more accurate picture of their morphology and properties (e.g., Shepherd \& Churchwell 1996a,b; Zhang et al. 2001; Shepherd 2005). In recent years, there has been a growing interest in the young stellar objects (YSOs) located at the lower and the higher ends of the stellar mass spectrum. Less

^ OVRO data as FITS files are also available in electronic form at the CDS via anonymous ftp to cdsarc.u-strasbg.fr(130.79.128.5) or via http://cdsweb.u-strasbg.fr/cgi-bin/qcat?]/A+A/481/93 attention has been paid to intermediate-mass protostars, with masses in the range $2 M_{\odot} \leq M_{\star} \leq 10 M_{\odot}$, which can power energetic molecular outflows. In fact, only a few deeply embedded intermediate-mass protostars are known to date, and only a very limited number of their outflows have been studied at high angular resolution.

To study in detail intermediate-mass protostars and the outflows that they are powering and to compare their morphology and evolution with those of low-mass stars, we started a project to carry out interferometric observations of dust and gas towards regions where intermediate-mass stars are being formed; hereafter intermediate-mass star-forming regions. The first two regions observed were IRAS 21391+5802 (Beltrán et al. 2002, hereafter Paper I) and L1206 (Beltrán et al. 2006b, hereafter Paper II). In this third paper, we present a thorough interferometric study of the intermediate-mass protostar IRAS 20050+2720 and of its molecular outflow. IRAS $20050+2720$ is a $280 L_{\odot}$ (Froebrich 2005) YSO located in the Cygnus rift at a distance 
Table 1. Parameters of the observations.

\begin{tabular}{lcccccc}
\hline \hline & & \multicolumn{2}{c}{ Synthesized beam } & & \\
Observation & Telescope & $\begin{array}{c}\text { Frequency } \\
(\mathrm{GHz})\end{array}$ & $\begin{array}{c}\text { HPBW } \\
(\operatorname{arcsec})\end{array}$ & $\begin{array}{c}\text { PA } \\
(\mathrm{deg})\end{array}$ & $\begin{array}{c}\text { Spectral resolution } \\
\left(\mathrm{km} \mathrm{s}^{-1}\right)\end{array}$ & $\begin{array}{c}\text { rms noise }^{a} \\
\left(\mathrm{mJy} \mathrm{beam}^{-1}\right)\end{array}$ \\
\hline $2.7 \mathrm{~mm}$ continuum & OVRO & 111.02 & $7.0 \times 6.4$ & -49 & - & 1.5 \\
$\mathrm{CO}(J=1 \rightarrow 0)$ & OVRO & 115.27 & $6.8 \times 4.1$ & -59 & 0.65 & 55 \\
$\mathrm{C}^{18} \mathrm{O}(J=1 \rightarrow 0)$ & OVRO & 109.78 & $8.1 \times 6.0$ & -43 & 0.68 & 40 \\
$\mathrm{HC}_{3} \mathrm{~N}(J=12 \rightarrow 11)$ & OVRO & 109.17 & $8.1 \times 6.0$ & -47 & 0.69 & 40 \\
$7 \mathrm{~mm}$ continum & VLA & 43.34 & $4.9 \times 3.8$ & -9 & - & 0.16 \\
\hline
\end{tabular}

${ }^{a}$ For the molecular line observations the $1 \sigma$ noise is per channel.

of 700 pc (Dame \& Thaddeus 1985). Distances in the Cygnus region are highly uncertain for stellar objects located at Galactic longitudes close to $90^{\circ}$, as the kinematic distances are not reliable (e.g. Schneider et al. 2006). However, IRAS 20050+2720 has a Galactic longitude of $\sim 66^{\circ}$, since located in a region where the Cygnus rift can be distinguished from the Cygnus X complex. Therefore, the kinematic distance determined by Dame \& Thaddeus (1985) from CO line emission is quite reliable. IRAS $20050+2720$ is surrounded by a cluster of infrared stars (Chen et al. 1997). Bachiller et al. (1995) mapped a high-velocity multipolar $\mathrm{CO}$ outflow in the region, previously detected by Wilking et al. (1989). IRAS $20050+2720$ is deeply embedded in a core that has been observed in the continuum at millimeter (Wilking et al. 1989; Chen et al. 1997; Choi et al. 1999; Chini et al. 2001; Furuya et al. 2005; Beltrán et al. 2006a) and centimeter (Wilking et al. 1989; Anglada et al. 1998) wavelengths and also in different high-density tracers, such as $\mathrm{HCO}^{+}$and $\mathrm{HCN}$ (Choi et al. 1999), $\mathrm{NH}_{3}$ (Molinari et al. 1996), CS and $\mathrm{N}_{2} \mathrm{H}^{+}$(Bachiller et al. 1995; Williams \& Myers 1999), and ${ }^{13} \mathrm{CO}$ and $\mathrm{C}^{18} \mathrm{O}$ (Ridge et al. 2003). The source has been classified as an intermediate-mass Class 0/I protostar (Gregersen et al. 1997; Chini et al. 2001; Froebrich 2005).

As shown by Papers I and II, intermediate-mass outflows appear more collimated and less complex than previously thought (e.g. Fuente et al. 2001) when observed with high-angular resolution. This argues for the need of high-angular observations to better understand intermediate-mass protostars and their outflows. To accomplish the ultimate goal of studying and comparing the properties and evolution of intermediate-mass protostars with those of their lower mass counterparts, in this last paper of the series, we put the results on IRAS 21391+5802, L1206, and IRAS $20050+2720$ in the context of intermediate-mass star formation. To do this, we have compiled information on outflow properties that have been derived from interferometric observations to better compare them with the ones derived for the sources in our study.

\section{Observations}

\subsection{OVRO observations}

Millimeter interferometric observations of IRAS 20050+2720 at $2.7 \mathrm{~mm}$ were carried out with the Owens Valley Radio Observatory (OVRO) Millimeter Array of six 10.4 m telescopes, in the L (Low) and C (Compact) configurations, on April 30 and May 30, 2003, respectively. The data taken in both array configurations were combined, resulting in baselines ranging from 5.6 to $42.6 \mathrm{k} \lambda$, which provided sensitivity to spatial structures from about $4 . .8$ to $37^{\prime \prime}$. The digital correlator was configured to simultaneously observe the continuum emission and some molecular lines. Details of the observations are given in Table 1 . The phase center was located at $\alpha(\mathrm{J} 2000)=20^{\mathrm{h}} 07^{\mathrm{h}} 5.80$,
$\delta(\mathrm{J} 2000)=+27^{\circ} 28^{\prime} 58^{\prime \prime}$. 1 . Bandpass calibration was achieved by observing the quasars $3 \mathrm{C} 84,3 \mathrm{C} 273$, and 3C 345 . Amplitude and phase were calibrated by observations of the nearby quasar J2015+71, whose flux density was determined relative to Uranus. The uncertainty in the amplitude calibration is estimated to be $\sim 20 \%$. The OVRO primary beam is $\sim 64^{\prime \prime}$ (FWHM) at $115.27 \mathrm{GHz}$. The data were calibrated using the MMA package developed for OVRO (Scoville et al. 1993). Reduction and analysis of the data were carried out using standard procedures in the MIRIAD, AIPS, and GILDAS software packages. We subtracted the continuum from the line emission directly in the $(u, v)$ domain for $\mathrm{C}^{18} \mathrm{O}(J=1 \rightarrow 0)$ and $\mathrm{HC}_{3} \mathrm{~N}(J=12 \rightarrow 11)$ and in the image domain for $\mathrm{CO}(J=1 \rightarrow 0)$.

\subsection{VLA observations}

The interferometric observations at $7 \mathrm{~mm}$ were carried out on January 5, 2002 using the Very Large Array (VLA) of the National Radio Astronomy Observatory (NRAO) ${ }^{1}$ in the D configuration. The baselines ranged from 5.4 to $140 \mathrm{k} \lambda$, which provided sensitivity to spatial structures from about 1 ."5 to $37^{\prime \prime}$. The phase center was set to the position $\alpha(\mathrm{J} 2000)=20^{\mathrm{h}} 07^{\mathrm{h}} 5^{\mathrm{s}} .91$, $\delta(\mathrm{J} 2000)=+27^{\circ} 28^{\prime} 58^{\prime \prime} .2$. Absolute flux calibration was achieved by observing 3C286, with an adopted flux density of $1.45 \mathrm{Jy}$. Amplitude and phase were calibrated by observing $2023+318$, which has a bootstrapped flux of $0.48 \pm 0.01 \mathrm{Jy}$. CLEANed maps were made using the task IMAGR of the AIPS software of the NRAO, with the ROBUST parameter of Briggs (1995) set equal to 5 , which is close to natural weighting. We used a Gaussian taper in the $(u, v)$ domain when making the maps in order to enhance the extended emission in the region. The resulting synthesized beam is 4 .' $^{\prime} \times 3^{\prime \prime} .8$ at PA $=-9^{\circ}$, and the rms noise is $0.16 \mathrm{mJy}^{\text {beam }^{-1}}$.

\section{Results}

\subsection{Dust emission}

The maps of the millimeter continuum emission around the embedded source IRAS $20050+2720$ at 2.7 and $7 \mathrm{~mm}$ are shown in Fig. 1. The continuum emission at $2.7 \mathrm{~mm}$ has been resolved into three different sources, labeled OVRO 1, OVRO 2, and OVRO 3. OVRO 1 and OVRO 2 have also been detected at $7 \mathrm{~mm}$, but not OVRO 3, for which an upper limit $(3 \sigma)$ of $0.48 \mathrm{mJy} \mathrm{beam}^{-1}$ has been estimated. OVRO 3 is not as welldefined at $2.7 \mathrm{~mm}$ as the other two sources. However, the source is located within $\sim 1^{\prime \prime} .6$ of the near-infrared (NIR) source 5 of Chen et al. (1997; see discussion below about the correction to the positions given by these authors). The source is probably

\footnotetext{
1 NRAO is a facility of the National Science Foundation operated under cooperative agreement by Associated Universities, Inc.
} 


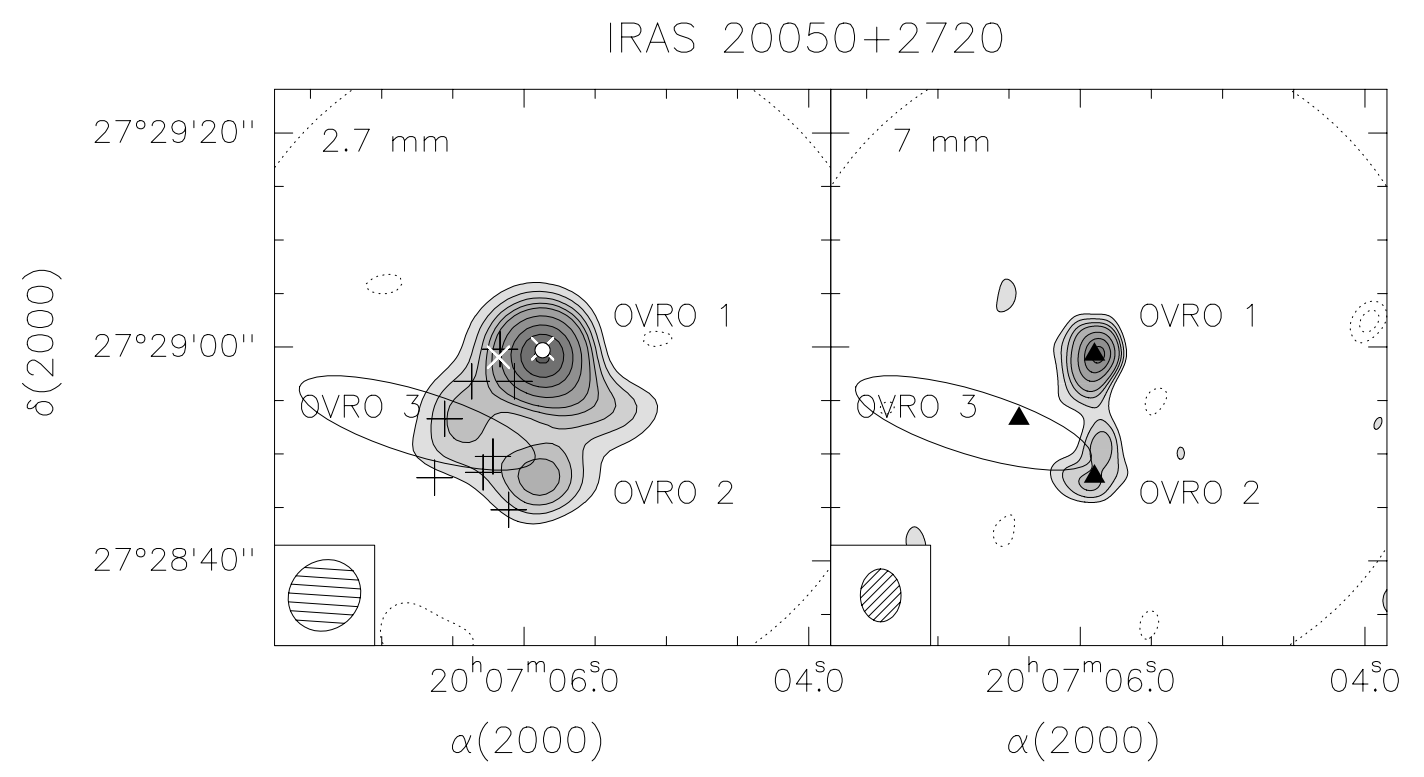

Fig. 1. Map of the OVRO $2.7 \mathrm{~mm}$ (left panel) and the VLA $7 \mathrm{~mm}$ (right panel) continuum emission towards IRAS 20050+2720. The rms noise of the map, $\sigma$, is $1.5 \mathrm{mJy}_{\text {beam }}^{-1}$ (left panel) and $0.16 \mathrm{mJy} \mathrm{beam}^{-1}$ (right panel). The contour levels are $-5,-3,3,5,7,9,11,15,20,25$, and 30 times $\sigma$ (left panel), and $-4,-3,3,4,5,6,8,10$, and 12 times $\sigma$ (right panel). The synthesized beams are drawn in the bottom left corner. The error ellipse of IRAS $20050+2720$ is indicated in black. The dotted circles represent the OVRO (left panel) and VLA (right panel) primary beam (50\% attenuation level). Black crosses indicate the corrected position (see Sect. 3.1) of the NIR sources detected by Chen et al. (1997), and white crosses the position of the $3 \mathrm{~mm}$ sources detected by Furuya et al. (2005). The white circle marks the position of the $3.6 \mathrm{~cm}$ VLA source reported by Anglada et al. (1998). Black triangles in the right panel mark the OVRO $2.7 \mathrm{~mm}$ continuum position of the sources.

Table 2. Positions, millimeter flux densities, sizes, and masses of the cores detected towards IRAS 20050+2720.

\begin{tabular}{|c|c|c|c|c|c|c|c|c|c|c|}
\hline \multirow[b]{2}{*}{ Core } & \multicolumn{2}{|c|}{ Position $^{a}$} & \multicolumn{2}{|c|}{$\lambda=2.7 \mathrm{~mm}$} & \multicolumn{2}{|c|}{$\lambda=7 \mathrm{~mm}$} & \multirow[b]{2}{*}{$\begin{array}{c}\theta^{c} \\
(\operatorname{arcsec})\end{array}$} & \multirow[b]{2}{*}{$\begin{array}{l}\operatorname{Mass}^{d} \\
\left(M_{\odot}\right)\end{array}$} & \multirow[b]{2}{*}{$\begin{array}{c}n\left(\mathrm{H}_{2}\right)^{e} \\
\left(10^{6} \mathrm{~cm}^{-3}\right)\end{array}$} & \multirow[b]{2}{*}{$\begin{array}{c}N\left(\mathrm{H}_{2}\right)^{e} \\
\left(10^{23} \mathrm{~cm}^{-2}\right)\end{array}$} \\
\hline & $\begin{array}{c}\alpha(\mathrm{J} 2000) \\
20^{\mathrm{h}} 07^{\mathrm{m}}\end{array}$ & $\begin{array}{l}\delta(\mathrm{J} 2000) \\
+27^{\circ} \quad 28^{\prime}\end{array}$ & $\begin{array}{c}I_{V}^{\text {peak } b} \\
\left(\mathrm{mJy} \mathrm{beam}^{-1}\right)\end{array}$ & $\begin{array}{c}S_{v}{ }^{b} \\
(\mathrm{mJy})\end{array}$ & $\begin{array}{c}I_{v}^{\text {peak } \quad b} \\
\left(\mathrm{mJy} \mathrm{beam}^{-1}\right)\end{array}$ & $\begin{array}{c}S_{v}{ }^{b} \\
(\mathrm{mJy})\end{array}$ & & & & \\
\hline OVRO 1 & $05^{\mathrm{s} .90}$ & $59 ! 3$ & $46.4 \pm 9.3$ & $65 \pm 13$ & $2.04 \pm 0.17$ & $2.88 \pm 0.17$ & 3.3 & 6.5 & 1.3 & 1.4 \\
\hline OVRO 2 & $05^{\mathrm{s}} .90$ & $47 . .9$ & $17.1 \pm 3.4$ & $18.0 \pm 3.6$ & $0.97 \pm 0.17$ & $1.84 \pm 0.17$ & 2.3 & 1.8 & 1.7 & 1.1 \\
\hline OVRO 3 & 06.43 & 53 ". 3 & $12.9 \pm 2.6$ & $12.9 \pm 2.6$ & $<0.48^{f}$ & $\ldots$ & $<6.7^{g}$ & $1.3^{h}$ & $3.0^{h}$ & $1.4^{h}$ \\
\hline
\end{tabular}

${ }^{a}$ Position of the $2.7 \mathrm{~mm}$ emission peak.

${ }^{b}$ Corrected for primary beam response.

${ }^{c}$ Deconvolved geometrical mean of the major and minor axes of the half-peak intensity contour.

${ }^{d}$ Circumstellar mass estimated taking into account all the emission inside the $3 \sigma$ level, and assuming a dust temperature of $34 \mathrm{~K}$ (see Sect. 4.1 ).

${ }^{e}$ Average $\mathrm{H}_{2}$ volume and column density estimated assuming spherical symmetry and a $10 \%$ helium abundance. The densities have been computed taking the total circumstellar mass and the size of the source at a $3 \sigma$ emission level into account.

$f 3 \sigma$ upper limit.

${ }^{g}$ Source not resolved.

${ }^{h}$ Estimated from the peak intensity.

associated with IRAS 20050+2720 (Fig. 1), at least at 12 and $25 \mu \mathrm{m}$ wavelengths. However, one cannot discard the possibility that part of the emission detected at longer wavelengths $(60$ and $100 \mu \mathrm{m}$ ) is associated with OVRO 1 and/or OVRO 2, as suggested by the IRAS high-resolution processed $\left(\mathrm{HIRES}^{2}\right)$ images. OVRO 3 is also associated with a $\mathrm{C}^{18} \mathrm{O}$ peak (see Sect. 3.3). All this led us to consider the bump visible at $2.7 \mathrm{~mm}$ emission as an independent source, which we called OVRO 3. OVRO 1 and OVRO 2 are separated $\sim 11^{\prime \prime}(\sim 8000 \mathrm{AU}$ at the distance of the region), while OVRO 3 is separated $\sim 9^{\prime \prime}$ ( $\left.\sim 6000 \mathrm{AU}\right)$ from the other two sources. The position, the flux density at $2.7 \mathrm{~mm}$, the deconvolved size of the sources (measured as the geometrical mean of the major and minor axes of the half-peak intensity

\footnotetext{
${ }^{2}$ HIRES images are available as single fields $\left(1^{\circ}-2^{\circ}\right)$ through the Infrared Processing and Analysis Center (IPAC).
}

contour around each source), the mass, and the average volume and column density are given in Table 2 .

The strongest millimeter source is OVRO 1. This source is located at the center of one of the two CO molecular outflows detected in the region (see the next section and Fig. 2), which suggests that OVRO 1 is its driving source. OVRO 1 has a core-halo structure at millimeter wavelengths, with a core that has a deconvolved diameter of $\sim 33^{\prime \prime} 3(\sim 2300 \mathrm{AU})$ at the half-peak intensity contour of the dust emission, and an extended and quite spherical halo or envelope that has a size of $\sim 11^{\prime \prime} 3(\sim 8000 \mathrm{AU})$ at the $3 \sigma$ contour level. The source OVRO 2 has a deconvolved diameter of $\sim 2$ !' $3(\sim 1600 \mathrm{AU})$ at the half-peak intensity contour of the dust emission. On the other hand, OVRO 3 is not resolved with the angular resolution of the $2.7 \mathrm{~mm}$ observations. These sizes are consistent with the values found for envelopes around lowand intermediate-mass protostars (e.g., Hogerheijde et al 1997, 1999; Looney et al. 2000; Fuente et al. 2001; Paper I; Paper II). 


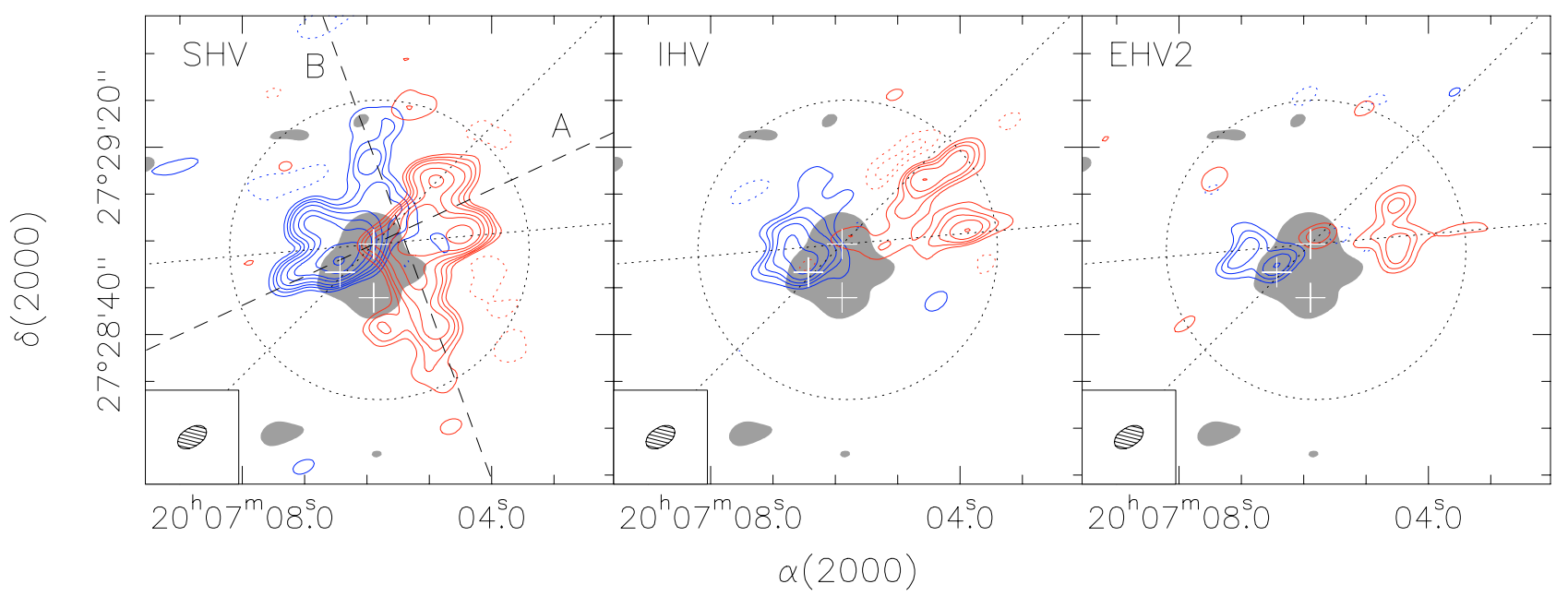

Fig. 2. $\mathrm{CO}(J=1 \rightarrow 0)$ emission integrated in different velocity intervals, $(-7,+1) \mathrm{km} \mathrm{s}^{-1}$ for the SHV blueshifted emission (left panel; blue contours), $(11,19) \mathrm{km} \mathrm{s}^{-1}$ for the redshifted one (left panel; red contours), $(-14,-7) \mathrm{km} \mathrm{s}^{-1}$ for the IHV blueshifted emission (middle panel; blue contours), and $(+19,+26) \mathrm{km} \mathrm{s}^{-1}$ for the redshifted one (middle panel; red contours), $(-19,-14) \mathrm{km} \mathrm{s}^{-1}$ for the EHV2 blueshifted emission (right panel; blue contours), and $(+26,+31) \mathrm{km} \mathrm{s}^{-1}$ for the redshifted one (right panel; red contours), overlaid on the $2.7 \mathrm{~mm}$ continuum emission at a $3 \sigma$ level (greyscale). Contour levels are $-5,-3,3,5,7,10,15,20,30$, and 40 times $\sigma$, where $1 \sigma$ is $0.6 \mathrm{Jy} \mathrm{beam}^{-1} \mathrm{~km} \mathrm{~s}^{-1}$ for the SHV interval, $0.5 \mathrm{Jy}$ beam ${ }^{-1} \mathrm{~km} \mathrm{~s}^{-1}$ for IHV, and $0.25 \mathrm{Jy}_{\text {beam }}^{-1} \mathrm{~km} \mathrm{~s}^{-1}$ for EHV2. The white crosses mark the millimeter continuum positions of OVRO 1 , OVRO 2, and OVRO 3. The black dashed lines in left panel outline the direction of outflows A, and B (see Sect. 3.2). The black dotted lines outline the edges of outflow A powered by OVRO 1. The synthesized beam is drawn in the bottom left corner. The dotted circle represents the OVRO primary beam (50\% attenuation level).

As can be seen in Fig. 1, there is extended emission surrounding the three sources, which is clearly visible at $2.7 \mathrm{~mm}$ but not at $7 \mathrm{~mm}$. The estimated upper limit $(3 \sigma)$ for the extended emission at $7 \mathrm{~mm}$ is $0.48 \mathrm{mJy}_{\text {beam }}{ }^{-1}$. This extended component has a size of $\sim 19^{\prime \prime}(\sim 13000 \mathrm{AU})$.

The position of the eight NIR sources detected by Chen et al. (1997) are indicated in Fig. 1. We compared these positions with those of the 2MASS catalog and found that the positions reported by Chen et al. (1997) were systematically offset on average by $\sim-0.29$ in right ascension, and $\sim 1^{\prime \prime} .4$ in declination. Therefore, before plotting them, we corrected the positions. As already mentioned, the only NIR source possibly associated with the millimeter sources detected with OVRO at $2.7 \mathrm{~mm}$ is source 5, whose position nearly coincides with OVRO 3. Chen et al. (1997) also detect a compact source at $2.7 \mathrm{~mm}$ with the Berkeley-Illinois-Maryland Association (BIMA) array coincident with OVRO 1. These authors estimate a flux density of $30 \pm 3.5 \mathrm{mJy}$, which is half of what is derived with OVRO (see Table 2). Although Chen et al. (1997) locate the millimeter continuum source close to the NIR sources 6,7 , and 8 , this is not the case after correcting the infrared positions. Therefore, this suggests that OVRO 1 and OVRO 2 could be deeply embedded sources still undetected at NIR wavelengths. The situation could be different for OVRO 3 because of its possible association with IRAS $20050+2720$ and NIR source 5 .

The region has also been observed at centimeter wavelengths with the VLA by Anglada et al. (1998). As can be seen in Fig. 1, the source OVRO 1 coincides within 0.5 with a $3.6 \mathrm{~cm}$ source, with two components separated by $\sim 0$ '.3 (Anglada et al. 1998).

The position of the millimeter source detected by Furuya et al. (2005) with the OVRO interferometer at $3 \mathrm{~mm}$ is indicated in Fig. 1 and is clearly coincident with OVRO 1. These authors also find millimeter emission eastward of OVRO 1, which could be associated with one of the NIR sources. This suggests that another YSO could be embedded in the core (Furuya et al. 2005). The position of this possible embedded YSO detected by Furuya et al. (2005) is also marked in Fig. 1. Our observations do not have enough resolution to separate this eastern emission from that of OVRO 1 and its extended envelope. The total integrated flux density measured by Furuya et al. (2005) at $3 \mathrm{~mm}$ is $25.6 \mathrm{mJy}$. If we assume a dust absorption coefficient proportional to $v^{2}$, the expected flux at $2.7 \mathrm{~mm}$ would be $\sim 39 \mathrm{mJy}$, which is about half of the integrated flux density measured for OVRO 1 at $2.7 \mathrm{~mm}$. This is probably due to the Furuya et al. observations being carried out with more extended OVRO configurations ( $\mathrm{E}$ and $\mathrm{H}$ ) and thus being somewhat less sensitive to extended emission. As a consequence of this, it is possible that part of the extended emission surrounding OVRO 1 was filtered out by the interferometer, consistent with the negative contours visible in Fig. 1 of Furuya et al. (2005).

Choi et al. (1999) observed the region with BIMA at $3.4 \mathrm{~mm}$, with a synthesized beam slightly larger than that of our $2.7 \mathrm{~mm}$ OVRO observations, and resolve the continuum emission into four sources, labeled A, B, C, and D. The peak position of source A agrees with OVRO 1. This source is extended and elongated towards the south and the southeast, and its structure resembles that of the extended emission surrounding OVRO 1, 2, and 3 as mapped by us at $2.7 \mathrm{~mm}$.

Chini et al. (2001) observed the region with the singledish James Clerk Maxwell Telescope (JCMT) at 0.45, 0.85, and $1.3 \mathrm{~mm}$, with angular resolutions ranging from $\sim 8$ to $15^{\prime \prime}$, and detected three sources in the region, IRAS 20050+2720 MMS 1, MMS 2, and MMS 3, together with an extension to the south of MMS 1. The source IRAS 20050+2720 MMS 1 is associated with OVRO 1, 2, and 3. Beltrán et al. (2006a) detect a similar emission with the Swedish-ESO Submillimetre Telescope (SEST) at $1.3 \mathrm{~mm}$. The clump number 1 is the one associated with OVRO 1, 2, and 3. However, this clump, which has a size of $58^{\prime \prime} .2$, is much larger than the extended $2.7 \mathrm{~mm}$ emission or MMS 1, as it includes the extended southern emission as well.

\subsection{The molecular outflow: $\mathrm{CO}$ emission}

The CO emission towards IRAS $20050+2720$ has been previously studied through lower angular resolution observations 


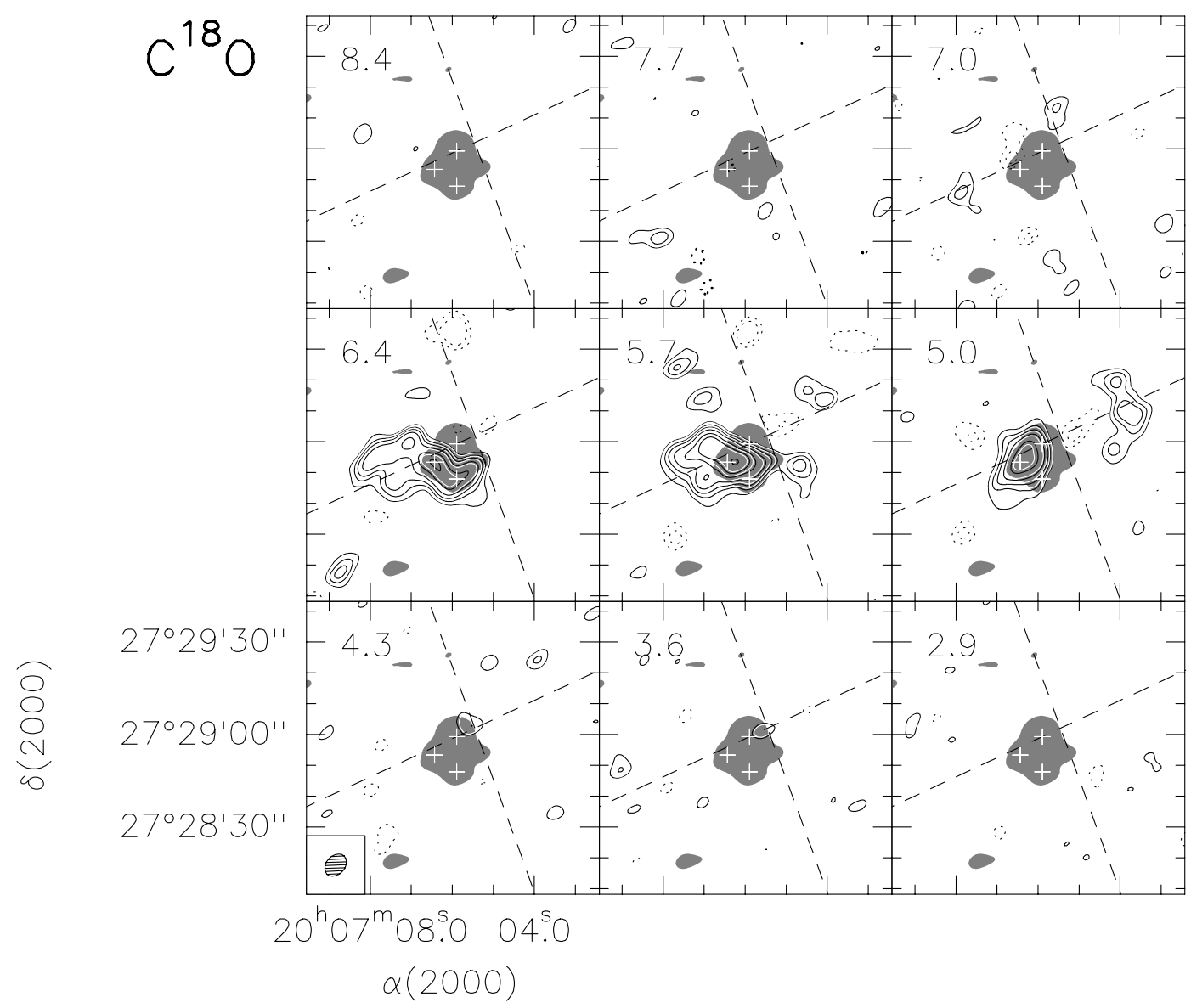

Fig. 3. Velocity channel maps of the $\mathrm{C}^{18} \mathrm{O}(J=1 \rightarrow 0)$ emission (contours) overlaid on the $2.7 \mathrm{~mm}$ continuum emission at a $3 \sigma$ level (greyscale). The systemic velocity of IRAS $20050+2720$ is $6 \mathrm{~km} \mathrm{~s}^{-1}$. The central velocity of each channel is indicated in the upper left corner of the panels. The $1 \sigma$ noise in one channel is $40 \mathrm{mJy}_{\text {beam }}{ }^{-1}$. Contour levels are $-5,-3,3,5,7,10,15,20$, and 30 times $\sigma$. The conversion factor from Jy beam ${ }^{-1}$ to $\mathrm{K}$ is 2.10 . The synthesized beam is drawn in the bottom left corner of the bottom left panel. The white crosses mark the millimeter continuum positions of OVRO 1, OVRO 2, and OVRO 3. The dashed lines outline the direction of the CO outflows A and B detected in the region (see Fig. 2).

Table 3. $\mathrm{C}^{18} \mathrm{O}(J=1 \rightarrow 0)$, and $\mathrm{HC}_{3} \mathrm{~N}(J=12 \rightarrow 11)$ line and physical parameters towards OVRO 1, OVRO 2, and OVRO 3 in IRAS $20050+2720$.

\begin{tabular}{llcccccr}
\hline \hline Position & Molecule & $\begin{array}{c}V_{\mathrm{LSR}} \\
\left(\mathrm{km} \mathrm{s}^{-1}\right)\end{array}$ & $\begin{array}{c}\Delta V \\
\left(\mathrm{~km} \mathrm{~s}^{-1}\right)\end{array}$ & $\begin{array}{c}T_{\mathrm{B}} \\
(\mathrm{K})\end{array}$ & $\begin{array}{c}T_{\mathrm{B}} \mathrm{d} V \\
\left(\mathrm{~K} \mathrm{~km} \mathrm{~s}^{-1}\right)\end{array}$ & $\begin{array}{c}\theta^{a} \\
(\operatorname{arcsec})\end{array}$ & $\begin{array}{c}M_{\text {vir }}^{b} \\
\left(M_{\odot}\right)\end{array}$ \\
\hline OVRO 1 & $\mathrm{C}^{18} \mathrm{O}$ & $4.68 \pm 0.04$ & $1.50 \pm 0.21$ & $0.94 \pm 0.11$ & $1.50 \pm 0.07$ & & \\
& $\mathrm{HC}_{3} \mathrm{~N}$ & $4.82 \pm 0.02$ & $1.44 \pm 0.05$ & $3.80 \pm 0.12$ & $5.84 \pm 0.18$ & $9 .{ }^{\prime} 6$ & $4.3-5.7$ \\
\hline OVRO 2 & $\mathrm{C}^{18} \mathrm{O}$ & $6.33 \pm 0.04$ & $1.70 \pm 0.10$ & $1.56 \pm 0.19$ & $2.83 \pm 0.12$ & $7^{\prime \prime} 0^{c}$ & $<4.3-5.8$ \\
& $\mathrm{HC}_{3} \mathrm{~N}$ & $5.86 \pm 0.08$ & $1.60 \pm 0.20$ & $0.85 \pm 0.13$ & $1.44 \pm 0.17$ & & \\
\hline OVRO 3 & $\mathrm{C}^{18} \mathrm{O}$ & $5.61 \pm 0.03$ & $1.92 \pm 0.06$ & $2.51 \pm 0.17$ & $5.14 \pm 0.15$ & 14 & $11-15$ \\
& $\mathrm{HC}_{3} \mathrm{~N}$ & $5.41 \pm 0.03$ & $1.30 \pm 0.10$ & $2.06 \pm 0.14$ & $2.85 \pm 0.14$ & & \\
\hline
\end{tabular}

${ }^{a}$ Deconvolved geometrical mean of the major and minor axes of the half-peak intensity contour.

${ }^{b}$ Estimated from Eq. (5) of Paper II for typical density distributions with $p=2.0-1.5$.

${ }^{c}$ Emission not resolved. Beam size.

by Bachiller et al. (1995). These authors discover three bipolar molecular outflows in the region: an extremely high-velocity, highly collimated outflow oriented nearly east-west (labeled A in Fig. 3 of Bachiller et al.); a bipolar outflow of intermediate high velocity oriented in the northeast-southwest direction (labeled B); and a standard high-velocity bipolar outflow detected along the northwest-southeast direction (labeled C). The observations reported in the present study significantly improve the angular resolution of previous outflow maps, revealing the structure of the molecular outflows in detail, as the interferometer filters out most of the extended $\mathrm{CO}$ emission seen in the Bachiller et al. (1995) low and intermediate velocity maps.
Figure 2 shows the maps of the integrated $\mathrm{CO}(J=1 \rightarrow 0)$ emission in three different blueshifted and redshifted velocity intervals. For the sake of comparison, we chose the same velocity intervals as Bachiller et al. (1995): a standard highvelocity (SHV) interval, with $(-7,+1) \mathrm{km} \mathrm{s}^{-1}$ for blueshifted emission and $(11,19) \mathrm{km} \mathrm{s}^{-1}$ for the redshifted one; an intermediate high-velocity (IHV) interval, with $(-14,-7) \mathrm{km} \mathrm{s}^{-1}$ for the blueshifted emission and $(+19,+26) \mathrm{km} \mathrm{s}^{-1}$ for the redshifted one; and an extremely high-velocity (EHV2) interval, with ( -19 , $-14) \mathrm{km} \mathrm{s}^{-1}$ for the blueshifted emission and $(+26,+31) \mathrm{km} \mathrm{s}^{-1}$ for the redshifted one. The other extremely high-velocity interval, EHV1, observed by Bachiller et al. (1995) was not mapped 


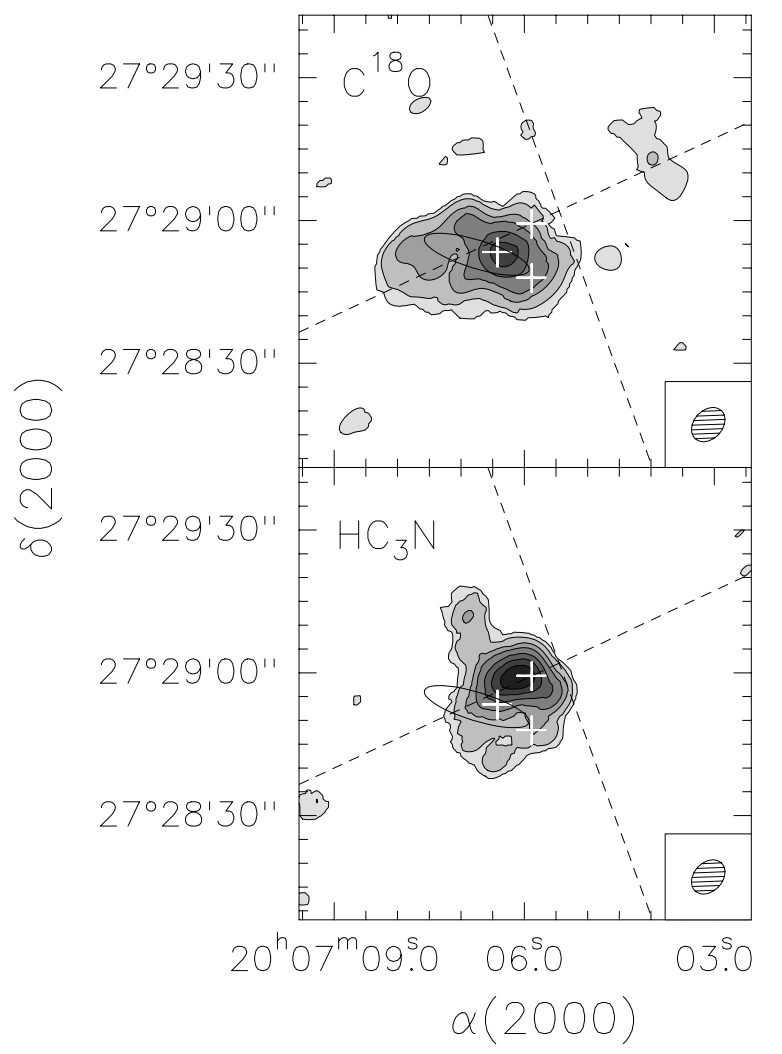

Fig. 4. Integrated intensity map of the $\mathrm{C}^{18} \mathrm{O}(J=1 \rightarrow 0)$ (top) and the $\mathrm{HC}_{3} \mathrm{~N}(J=12 \rightarrow 11)$ (bottom) emission over the velocity interval $(3,8) \mathrm{km} \mathrm{s}^{-1}$ towards IRAS 20050+2720. Contour levels are 0.17, 0.34, $0.68,1.0,1.5,2.0$, and $2.5 \mathrm{Jy}^{\text {beam }}{ }^{-1} \mathrm{~km} \mathrm{~s}^{-1}$ for both panels. The white crosses show the millimeter continuum positions of OVRO 1, OVRO 2, and OVRO 3. The dashed lines indicate the direction of the $\mathrm{CO}$ outflows A and B. The error ellipse of IRAS $20050+2720$ is indicated in black. The synthesized beam is drawn in the bottom right corner of each panel.

because our OVRO observations did not cover that range of velocities. The systemic velocity, $V_{\mathrm{LSR}}$, is roughly $6 \mathrm{~km} \mathrm{~s}^{-1}$. As can be seen in this figure, the $\mathrm{CO}$ emission traces two bipolar outflows in the region, a roughly east-west outflow, coincident with outflow A of Bachiller et al. (1995), and a northeast-southwest outflow, coincident with outflow B. The outflow labeled $\mathrm{C}$ by Bachiller et al. (1995) and mapped at the lowest velocity interval (SHV) was not detected. As can be seen in Fig. 3 of Bachiller et al. (1995), the outflow $\mathrm{C}$, which has a PA of about $-35^{\circ}$, has very extended lobes, especially for the blueshifted emission that reaches distances of $>100^{\prime \prime}$ from the IRAS source. Most of the emission of this outflow lies outside of the OVRO primary beam and, therefore, these interferometric observations are not sensitive to it. Note that one cannot rule out the possibility that part of the central emission of the outflow $\mathrm{C}$ overlaps that of the outflows $\mathrm{A}$ and $\mathrm{B}$; and as it is not possible to distinguish any possible emission of the outflow $\mathrm{C}$ from that of the outflows $\mathrm{A}$ and $\mathrm{B}$, we considered the outflow $\mathrm{C}$ as not detected in this study.

\section{3. $\mathrm{C}^{18} \mathrm{O}$ emission}

Figure 3 shows the velocity channel maps for the $\mathrm{C}^{18} \mathrm{O}(J=1 \rightarrow 0)$ emission around the systemic velocity, $V_{\mathrm{LSR}} \simeq 6 \mathrm{~km} \mathrm{~s}^{-1}$, overlaid on the continuum emission. The emission integrated over the central channels, velocity interval $(3,8) \mathrm{km} \mathrm{s}^{-1}$, is shown in the top panel of Fig. 4. The emission
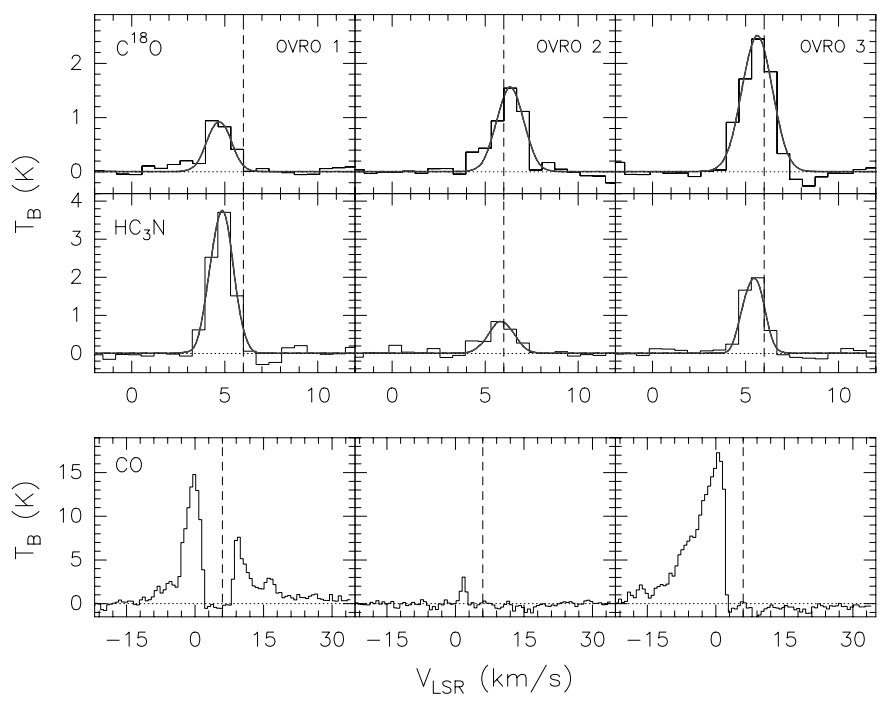

Fig. 5. $\mathrm{C}^{18} \mathrm{O}(\mathrm{J}=1 \rightarrow 0)$ (top), $\mathrm{HC}_{3} \mathrm{~N}(J=12 \rightarrow 11)$ (middle), and $\mathrm{CO}(J=1 \rightarrow 0)$ (bottom) spectra obtained at the position of OVRO 1 , OVRO 2, and OVRO 3 in IRAS 20050+2720. The continuum has been subtracted. The conversion factor is $2.10 \mathrm{~K} / \mathrm{Jy}$ beam ${ }^{-1}$ for $\mathrm{C}^{18} \mathrm{O}$, $2.14 \mathrm{~K} / \mathrm{Jy} \mathrm{beam}^{-1}$ for $\mathrm{HC}_{3} \mathrm{~N}$, and $3.30 \mathrm{~K} / \mathrm{Jy}_{\text {beam }}{ }^{-1}$ for $\mathrm{CO}$. The thick grey profiles in top and middle panels are the Gaussian fits to the spectra. The dashed vertical line indicates the systemic velocity of $6 \mathrm{~km} \mathrm{~s}^{-1}$.

is elongated in the east-west direction and peaks towards OVRO 3. The $\mathrm{C}^{18} \mathrm{O}$ emission is more extended than the dust one. In particular, it shows an elongation in the direction of the molecular outflow A, which suggests some interaction between the outflow and the envelope. The spectra taken at the position of the continuum peak of OVRO 1, OVRO 2, and OVRO 3 are shown in Fig. 5. Table 3 lists the fitted parameters for $\mathrm{C}^{18} \mathrm{O}$ $(J=1 \rightarrow 0)$ and $\mathrm{HC}_{3} \mathrm{~N} \quad(J=12 \rightarrow 11)$. The corresponding Gaussian fits are shown in Fig. 5. As can be seen in the channel maps and the spectra, the $\mathrm{C}^{18} \mathrm{O}$ emission is associated with the three millimeter sources, with the emission towards OVRO 1 weaker than towards OVRO 2 and 3. The velocity-integrated flux density of the central emission is $\sim 16 \mathrm{Jy} \mathrm{km} \mathrm{s}^{-1}$ and has a deconvolved size of $\sim 14^{\prime \prime}$ or $\sim 9800 \mathrm{AU}$, measured as the geometrical mean of the major and minor axes of the half-peak intensity contour of the gas.

\section{4. $\mathrm{HC}_{3} \mathrm{~N}$ emission}

Figure 6 shows the velocity channel maps for the $\mathrm{HC}_{3} \mathrm{~N}(J=12 \rightarrow 11)$ emission towards IRAS 20050+2720, around the systemic velocity, $V_{\mathrm{LSR}} \simeq 6 \mathrm{~km} \mathrm{~s}^{-1}$, overlaid on the continuum emission. The emission integrated over the velocity interval $(3,8) \mathrm{km} \mathrm{s}^{-1}$ is shown in the bottom panel of Fig. 4. There, $\mathrm{HC}_{3} \mathrm{~N}$ shows a spatial distribution different from that of $\mathrm{C}^{18} \mathrm{O}$ and the dust. This is probably because the different molecular tracers and the dust are tracing material in slightly different physical conditions. The $\mathrm{C}^{18} \mathrm{O}$ elongation along outflow $\mathrm{A}$ is not traced by $\mathrm{HC}_{3} \mathrm{~N}$, which could indicate that the gas is not dense enough to excite the $\mathrm{HC}_{3} \mathrm{~N}(J=12 \rightarrow 11)$ transition. The spectra taken at the position of the continuum peak of OVRO 1 , OVRO 2, and OVRO 3 are shown in Fig. 5. The emission peaks about 4". 2 east of the continuum peak position of OVRO 1, and it is clearly associated with this source. The $\mathrm{HC}_{3} \mathrm{~N}$ emission is also associated with the other two sources in the region, with the emission towards OVRO 2 weaker than towards OVRO 1 


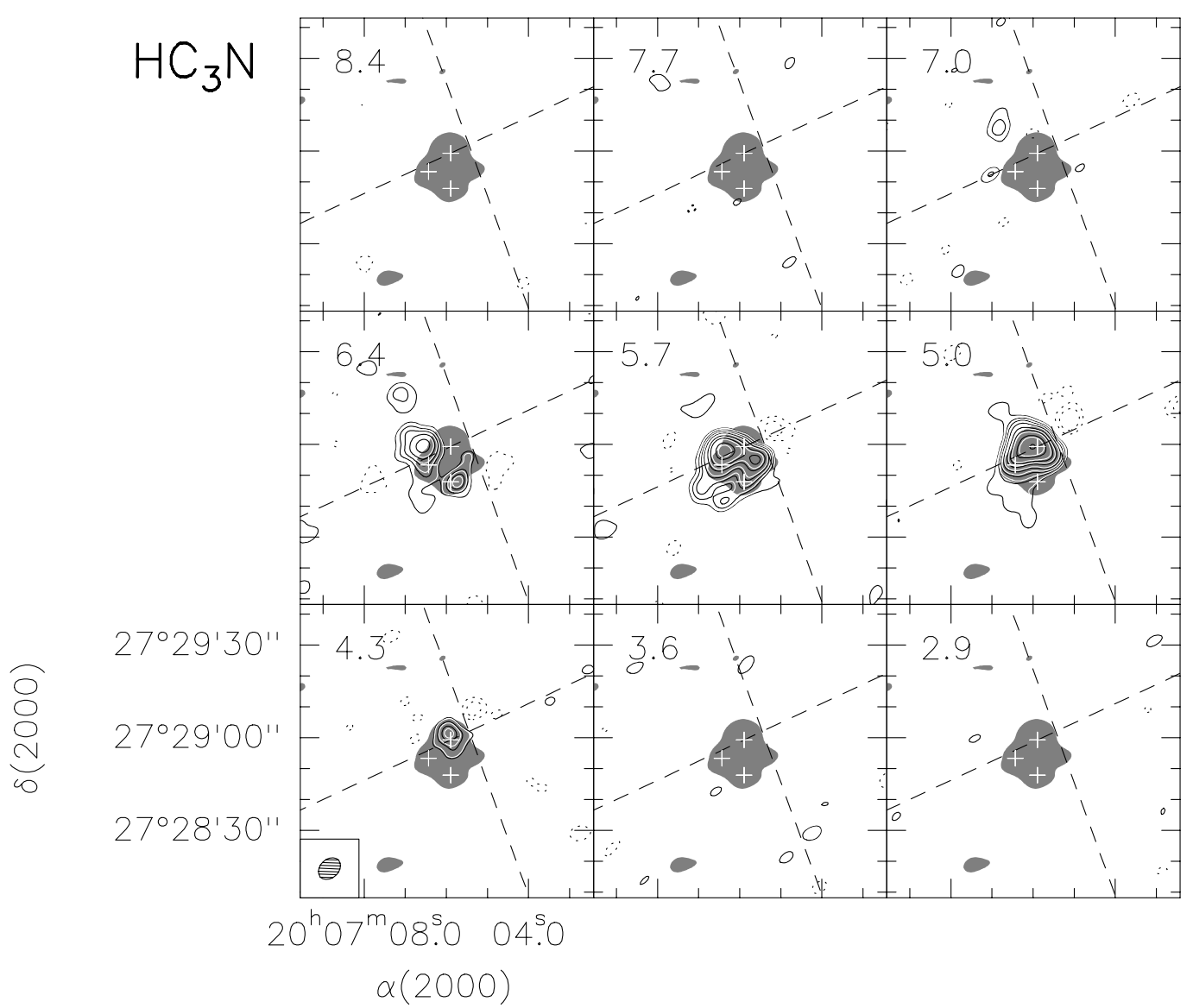

Fig. 6. Velocity channel maps of the $\mathrm{HC}_{3} \mathrm{~N}(J=12 \rightarrow 11)$ emission (contours) overlaid on the $2.7 \mathrm{~mm}$ continuum emission at a $3 \sigma$ level (greyscale). The systemic velocity of IRAS $20050+2720$ is $6 \mathrm{~km} \mathrm{~s}^{-1}$. The central velocity of each channel is indicated in the upper left corner of the panels. The $1 \sigma$ noise in one channel is $40 \mathrm{mJy} \mathrm{beam}^{-1}$. Contour levels are $-5,-3,3,5,7,10,15,20,30$, and 45 times $\sigma$. The conversion factor from Jy beam ${ }^{-1}$ to $\mathrm{K}$ is 2.14 . The synthesized beam is drawn in the bottom left corner of the bottom left panel. The white crosses mark the millimeter continuum positions of OVRO 1, OVRO 2, and OVRO 3. The dashed lines outline the direction of the CO outflows A and B (see Fig. 2).

and 3. Although the emission is quite compact, it shows a tail towards the northeast, and it is slightly elongated towards the southeast, along the direction of the molecular outflow A. The $\mathrm{HC}_{3} \mathrm{~N}$ emission has a velocity integrated flux density of $\sim 12.7 \mathrm{Jy} \mathrm{km} \mathrm{s}^{-1}$ and a deconvolved size of 9.' 6 or $\sim 6700 \mathrm{AU}$.

\section{Analysis and discussion of IRAS $20050+2720$}

\subsection{Mass and density estimates from dust emission}

Assuming that the dust emission is optically thin, we estimated the masses of the sources (Table 2) following Eq. (1) of Paper II and adopting a dust mass-opacity coefficient at $111 \mathrm{GHz}, \kappa_{111}=0.2 \mathrm{~cm}^{2} \mathrm{~g}^{-1}\left(\kappa_{0}=1 \mathrm{~cm}^{2} \mathrm{~g}^{-1}\right.$ at $250 \mathrm{GHz}$ : Ossenkopf \& Henning 1994) and a gas-to-dust ratio of 100. The dust temperature assumed to derive the masses is $34 \mathrm{~K}$, which is the dust temperature obtained by Chini et al. (2001) by fitting a grey-body to the IRAS fluxes at 60 and $100 \mu \mathrm{m}$ and the SCUBA fluxes at 450,850 , and $1300 \mu \mathrm{m}$. The mass estimated is $6.5 M_{\odot}$ for OVRO $1,1.8 M_{\odot}$ for OVRO 2 , and $1.3 M_{\odot}$ for OVRO 3. Note that using a lower mass-opacity coefficient as proposed by Hildebrand (1983) would result in masses higher by a factor of $\sim 4$, whereas $10 \mathrm{~K}$ lower temperatures would increase the masses by a factor of $\sim 1.5$. The circumstellar masses of the three deeply embedded sources are similar to those found for intermediate-mass YSOs for OVRO 1, and for low-mass protostars for OVRO 2 and 3. The average $\mathrm{H}_{2}$ volume density, $n\left(\mathrm{H}_{2}\right)$, and column density, $N\left(\mathrm{H}_{2}\right)$, of the sources estimated by assuming spherical symmetry are given in Table 2.

\subsection{The molecular outflows}

\subsubsection{Morphology}

The outflow A, which was detected at the three velocity intervals, is centered at the position of OVRO 1. It is elongated in the direction of $\mathrm{PA} \simeq 115^{\circ}$, and presents a different morphology for the blueshifted and the redshifted emission. The blueshifted emission is more compact than the redshifted one. This lack of symmetry between the lobes could be due to the presence of OVRO 3. In such a scenario, the redshifted gas would flow freely westwards of the powering source OVRO 1 , while the blueshifted gas, moving eastwards, would interact with the OVRO 3 core that in projection seems to be located in the direction of the blue lobe. The CO spectra towards OVRO 3 in Fig. 5 exhibits a high-velocity blueshifted wing that supports the "impact" hypothesis. Such a situation resembles the one in IRAS 21391+5802 (Paper I), where the molecular outflow interacts with the dense material in the surroundings and gets deflected. The blueshifted emission shows a lobe that shrinks as the velocity of the outflow increases, and the direction of the emission is roughly the same for all the velocity intervals. On the other hand, the redshifted emission is more extended and shows a more complex morphology. At standard (SHV) and 


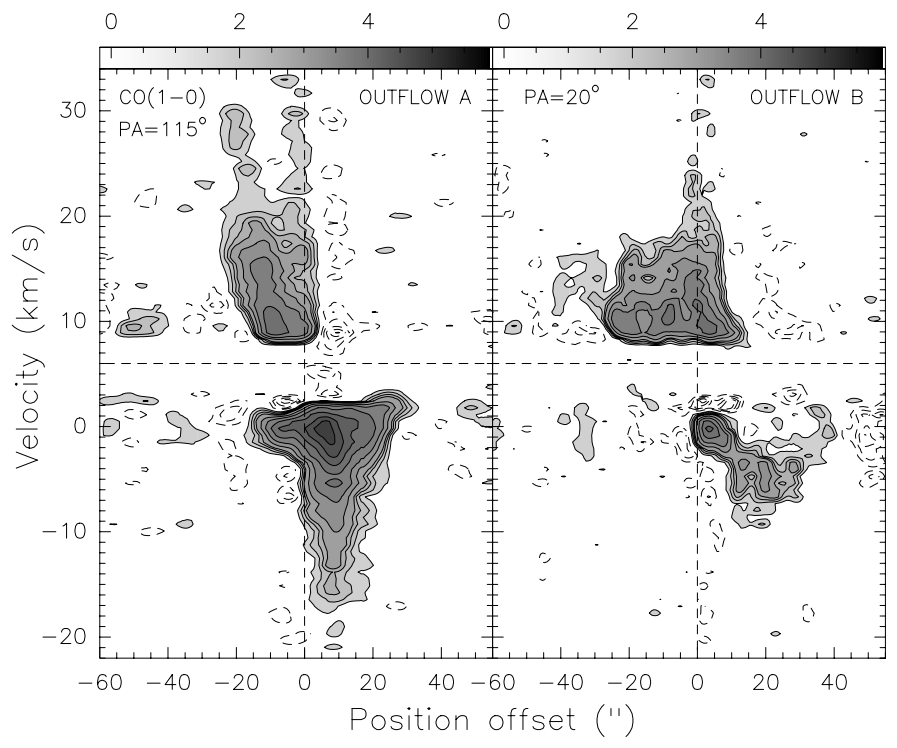

Fig. 7. PV plot of the $\mathrm{CO}(J=1 \rightarrow 0)$ emission along the major axis of the molecular outflow A with PA $=115^{\circ}$ (left), and of the outflow B with $\mathrm{PA}=20^{\circ}$ (right). The position offset is relative to the position of OVRO 1 (left) and relative to the center of symmetry of the outflow B, $\alpha(\mathrm{J} 2000)=20^{\mathrm{h}} 07^{\mathrm{h}} 05^{\mathrm{s}} 38, \delta(\mathrm{J} 2000)=+27^{\circ} 28^{\prime} 59^{\prime}{ }^{\prime} 3$. Contours are -1.2 , $-0.9,-0.72,-0.54,-0.36,-0.18,0.18,0.36,0.54,0.72,0.90,1.2,1.8$, $2.7,3.6,4.5$, and $5.4 \mathrm{Jy}_{\text {beam }}{ }^{-1}$. The horizontal line marks the systemic velocity, $V_{\mathrm{LSR}}=6 \mathrm{~km} \mathrm{~s}^{-1}$.

intermediate (IHV) outflow velocities, the emission seems to trace the walls of the cavity excavated by the outflow. As the velocity of the outflow increases, the angle of aperture of the conical cavity decreases, and the redshifted emission is detected at greater distances from OVRO 1. The farthest redshifted emission is located at $\sim 35^{\prime \prime}$ from OVRO 1 at the IHV interval. The CO emission is only detected at the center of the lobe at EHV2. In fact, at EHV2, the redshifted emission is not tracing the walls of the cavity. That no CO emission is detected inside the cavity for SHV and IHV indicates that the gas has already been evacuated. This suggests that the outflow and therefore the powering source OVRO 1 could be in a later Class 0 evolutionary stage. Figure 7 shows the position-velocity (PV) plot along the major axis of the outflow A and centered on the position of OVRO 1, and the PV plot along the major axis of the outflow $\mathrm{B}$ and centered on the center of symmetry of the outflow (see below). As can be seen in Fig. 7, there is blueshifted emission at negative offsets for the outflow A, and redshifted emission at positive offsets for the outflow B. This probably indicates that the two outflows overlap along the line-of-sight near their centers of symmetry where the powering sources are embedded or that the outflows are not resolved at the base by the current resolution.

The bipolar outflow B has a PA of $\sim 20^{\circ}$ and it has been clearly detected at SHV. At IHV, only some weak blueshifted emission towards the NE is visible. The powering source is probably located close to the center of symmetry of the lobes. However, although there is some extended $2.7 \mathrm{~mm}$ continuum emission towards that position, the angular resolution of our observations is not high enough to separate the emission of the source driving outflow B from that of the circumstellar envelope surrounding OVRO 1. There are several 2MASS infrared sources close to the center of symmetry of the outflow, although it is difficult to determine whether one of these could be the driving source. The emission of this outflow presents two well-defined and elongated lobes, which have an extension of $\sim 30^{\prime \prime}$.
Table 4. Properties of the CO outflow.

\begin{tabular}{lccccc}
\hline \hline Lobe & $\begin{array}{c}V^{a} \\
\left(\mathrm{~km} \mathrm{~s}^{-1}\right)\end{array}$ & $\begin{array}{c}M^{b} \\
\left(M_{\odot}\right)\end{array}$ & $\begin{array}{c}P^{c} \\
\left(M_{\odot} \mathrm{km} \mathrm{s}^{-1}\right)\end{array}$ & $\begin{array}{c}E^{c} \\
\left(10^{44} \mathrm{erg}\right)\end{array}$ & $\begin{array}{c}F_{\text {out }}^{c} \\
\left(M_{\odot} \mathrm{km} \mathrm{s}^{-1} \mathrm{yr}^{-1}\right)\end{array}$ \\
\hline \multicolumn{5}{c}{ OUTFLOW A } \\
\hline Blue & {$[-19,+1]$} & 0.10 & 0.99 & 1.05 & $2.8 \times 10^{-4}$ \\
Red $[+11,+31]$ & 0.10 & 1.05 & 1.32 & $2.5 \times 10^{-4}$ \\
Total & 0.20 & 2.04 & 2.37 & $5.3 \times 10^{-4}$ \\
\hline \multicolumn{5}{c}{ OUTFLOW B } \\
\hline Blue & {$[-14,+1]$} & 0.02 & 0.23 & 0.24 & $6.4 \times 10^{-5}$ \\
Red $[+11,+19]$ & 0.03 & 0.19 & 0.14 & $4.5 \times 10^{-5}$ \\
Total & 0.05 & 0.42 & 0.38 & $1.1 \times 10^{-4}$ \\
\hline
\end{tabular}

${ }^{a}$ Range of outflow velocities.

${ }^{b}$ Assuming an excitation temperature of $29 \mathrm{~K}$.

${ }^{c}$ Momenta and kinetic energies are calculated relative to the cloud velocity, which is taken to be $V_{\mathrm{LSR}}=6 \mathrm{~km} \mathrm{~s}^{-1}$. Velocity not corrected for inclination.

\subsubsection{Physical parameters of the $\mathrm{CO}$ outflows}

Assuming that the CO emission is in LTE and is optically thin, the mass of the gas associated with the outflows detected in the region was calculated by using Eq. (2) of Paper II. The CO emission is expected to be optically thin at high-outflow velocities (see Sect. 3.3.4 of Arce \& Goodman 2001). If CO is optically thick in portions of the flow, the correction for opacity should in any case be small. As the CO spectra are complex and exhibit prominent self-absorption in both interferometric (see Fig. 5) and single-dish observations (Bachiller et al. 1995; Zhang et al. 2005), it is difficult to derive the excitation temperature from the $\mathrm{CO}$ brightness temperature. Therefore, taking the assumption of LTE into account, we assumed that the excitation temperature is equal to the kinetic temperature of $29 \mathrm{~K}$ derived from $\mathrm{NH}_{3}$ observations by Molinari et al. (1996), a temperature slightly lower than the dust temperature derived by fitting the spectral energy distribution (SED) by Chini et al. (2001) but similar to the excitation temperature used for other intermediatemass molecular outflows. We assumed an $\left[\mathrm{H}_{2}\right] /[\mathrm{CO}]$ abundance ratio of $10^{4}$ (e.g. Scoville et al. 1986) and a $10 \%$ helium abundance. In Table 4 we give the mass, the momentum, kinetic energy, and momentum rate in the outflows, which were estimated following the procedures given by Scoville et al. (1986). We also report the range of outflow velocities for the outflows. Note that the momentum, kinetic energy, and momentum rate in the outflow have not been corrected for the (unknown) inclination angle, $i$, of the flow with respect to the plane of the sky. In the case of correcting for inclination, the velocities should be divided by $\sin i$, and the linear size of the lobes by $\cos i$. It is almost impossible to separate the emission of both outflows for the SHV interval, especially close to the center of symmetry of the outflows (see previous section). Therefore, it is possible that some emission from outflow B has been counted as part of outflow A and vice versa. As already mentioned in Paper II, due to some possible filtering of the emission by the interferometer, absorption of the emission by the ambient cloud, opacity effects, and the integration range chosen, the masses calculated should be considered as lower limits. The values of the mass, momentum, kinetic energy, and momentum rate are consistent with the values estimated from interferometric observations for other intermediate-mass molecular outflows (see Table 5). The dynamical timescales of the outflows, which have been estimated taking the ratio of the maximum separation of the outflow lobes and the terminal velocity of the outflow measured 
from the CO line wings, are 3000 and $5000 \mathrm{yr}$ for the blueshifted and redshifted lobes of outflow A, and 5000 and $7000 \mathrm{yr}$ for the blueshifted and redshifted lobes of outflow B.

\subsection{The dense cores}

\subsubsection{Physical parameters of the dense cores}

As can be seen in the spectra (Fig. 5) and the fitted parameters (Table 3), the gas towards OVRO 1 appears to be clearly at a different velocity, $V_{\mathrm{LSR}} \simeq 4.7 \mathrm{~km} \mathrm{~s}^{-1}$, than OVRO 2 and OVRO 3 , which have velocities of $V_{\mathrm{LSR}} \simeq 5.5-6 \mathrm{~km} \mathrm{~s}^{-1}$.

Following Eq. (5) of Paper II, we estimated the virial mass of the sources assuming a spherical clump with a power-law density distribution $\rho \propto r^{-p}$, with $p=2.0-1.5$, and neglecting contributions from the magnetic field and surface pressure (see Table 3). The virial masses were computed from $\mathrm{C}^{18} \mathrm{O}$, except for OVRO 1. For this source, it was computed from $\mathrm{HC}_{3} \mathrm{~N}$ because it peaks close to OVRO 1 and is clearly associated with it. For OVRO 2, the emission is not resolved, and therefore one can only estimate an upper limit. The values estimated for OVRO $1,4.3-5.7 M_{\odot}$, are slightly lower than the mass derived from the continuum, $6.5 M_{\odot}$. Taking into account that the mass of the central protostar has not been considered in this calculation, the total mass (circumstellar plus protostar) of OVRO 1 would be slightly higher than the virial mass. This would be consistent with the object undergoing collapse and consistent with accretion driving the outflow. For OVRO 2, from the upper limits computed, it is not possible to say whether the virial mass is higher or lower than the continuum mass. For OVRO 3, the virial mass estimated is clearly higher than the mass derived from the continuum. However, it is not possible to compare both masses because the $\mathrm{C}^{18} \mathrm{O}$ emission, from which the virial mass has been computed, is much more extended than the continuum emission, from which the circumstellar mass has been estimated.

\subsubsection{Envelope mass from gas emission}

As already mentioned in Paper II, the gas abundance relative to molecular hydrogen is the main uncertainty in the estimate of the mass of the molecular core. Therefore, instead of measuring the mass of the gas towards the protostars from the $\mathrm{C}^{18} \mathrm{O}$ or $\mathrm{HC}_{3} \mathrm{~N}$ emission, assuming a given fractional abundance of these species, it is better to estimate their fractional abundance from a comparison with the mass derived from the dust emission. We integrated the gas emission in the same area used to estimate the mass of the gas from the continuum dust emission and assumed that the mass towards the continuum sources derived from $\mathrm{C}^{18} \mathrm{O}$ and $\mathrm{HC}_{3} \mathrm{~N}$ is what is derived from the $2.7 \mathrm{~mm}$ continuum emission.

Following Eqs. (3) and (4) of Paper II, the estimated $\mathrm{C}^{18} \mathrm{O}(\mathrm{J}=1 \rightarrow 0)$ abundance relative to molecular hydrogen is $2.5 \times 10^{-8}$ towards OVRO 1 and $\sim 5 \times 10^{-8}$ towards OVRO 2 and OVRO 3. Typical $\mathrm{C}^{18} \mathrm{O}$ fractional abundances estimated towards molecular clouds are $1.7-4 \times 10^{-7}$ (Frerking et al. 1982; Kulesa et al. 2005). Therefore, even taking the uncertainties into account up to a factor of 5 introduced in the mass estimates by the different dust opacity laws used, the abundances derived towards OVRO 1, 2, and 3 are lower than the typical fractional abundances.

Regarding the $\mathrm{HC}_{3} \mathrm{~N}$ emission, the estimated abundance relative to molecular hydrogen is $1 \times 10^{-10}$ towards OVRO 1 , $6.5 \times 10^{-11}$ towards OVRO 2 , and $8.5 \times 10^{-11}$ towards OVRO 3 . The $\mathrm{HC}_{3} \mathrm{~N}$ abundance found in the literature can vary by up to 3 orders of magnitude, from $\sim 3 \times 10^{-11}$ (Vanden Bout et al. 1983) to $\sim 10^{-8}$ (de Vicente et al. 2000; Martín-Pintado et al. 2005). Therefore, the $\mathrm{HC}_{3} \mathrm{~N}$ abundances derived towards OVRO 1, and especially those derived towards OVRO 2 and 3, are in the lower end of the range of fractional abundances estimated toward molecular clouds.

\section{Intermediate-mass protostars and their outflows}

The present work, which is focused on the properties of IRAS $20050+2720$ and of its molecular outflow, is the third in a series of high-angular resolution studies devoted to intermediate-mass protostars and their outflows, including the study of IRAS 21391+5802 (Paper I) and IRAS 22272+6358A (Paper II). We attempt to put the results on IRAS 20050+2720 in the context of our previous results and in the context of intermediate-mass star formation. We further compare the properties and evolution of intermediate-mass protostars with those of their lower mass counterparts. To do that, we compiled from the literature information on the outflow properties of intermediate-mass stars. We restricted our search to outflow properties that have been derived from interferometric observations in order to be sensitive to the same spatial scales and to better compare the properties with those derived for the sources in our study. Our sample is therefore not very homogeneous, as very few intermediate-mass outflows have been observed at high-angular resolution. In any case, we have been able to compile data of a total of 14 intermediate-mass protostars, including the 3 sources studied by us.

\subsection{Multiplicity in intermediate-mass star-forming regions}

The millimeter continuum emission around IRAS 20050+2720 has been resolved into three millimeter sources (see Fig. 1): an intermediate-mass source, OVRO 1, and two low-mass sources, OVRO 2 and OVRO 3. In addition, the powering source of outflow B has not yet been detected at millimeter wavelengths, or its emission has not been resolved from the extended emission of the circumstellar envelope surrounding the intermediate-mass OVRO 1 (see Sect. 3.2). If one also takes into account the millimeter source detected by Furuya et al. (2005), located about 5" east of OVRO 1 and not resolved by our observations, the emission around IRAS $20050+2720$ would be associated, at least, with 5 YSOs. And, except for OVRO 3 that could be associated with IRAS $20050+2720$ and with the NIR source 5 detected by Chen et al. (1997; see Sect. 3.1), none of them seems to have been detected at infrared wavelengths yet. This situation very closely resembles the scenario around the intermediatemass sources IRAS $21391+5802$ in the bright-rimmed cloud IC $1396 \mathrm{~N}$, and IRAS $22272+6358 \mathrm{~A}$ in L1206, where the millimeter emission has been resolved into an intermediate-mass source surrounded by less massive and smaller objects (Paper I; Beltrán et al. 2004a; Fuente et al. 2007; Neri et al. 2007; Paper II). This suggests that intermediate-mass stars form in dense clustered environments. In other words, the formation of an isolated intermediate-mass star, if existing, appears rare. This is different from the low-mass case, where examples of protostars formed in relative isolation are known (e.g. L1157: Beltrán et al. 2004b; HH 211: Palau et al. 2006; or Bok globules: Laundhart et al. 1997). The crowded environments would explain the complexity of the immediate vicinity of intermediatemass protostars.

Intermediate-mass protostars are associated with small protoclusters of low-mass millimeter sources, some of them not 
Table 5. Properties of intermediate-mass outflows derived from interferometric observations.

\begin{tabular}{lcccccccc}
\hline \hline \multicolumn{1}{c}{ Outflow } & $\begin{array}{c}d \\
\mathrm{kpc})\end{array}$ & $\begin{array}{c}L \\
\left(L_{\odot}\right)\end{array}$ & $\begin{array}{c}M_{\text {env }} \\
\left(M_{\odot}\right)\end{array}$ & $\begin{array}{c}M_{\text {out }} \\
\left(M_{\odot}\right)\end{array}$ & $\begin{array}{c}F_{\text {obs }} \\
\left(M_{\odot} \mathrm{km} \mathrm{s}^{-1} \mathrm{yr}^{-1}\right)\end{array}$ & $\begin{array}{c}t_{\text {dyn }} \\
\left(10^{3} \text { yr }\right)\end{array}$ & $\begin{array}{c}\text { NIR or } \\
\mathrm{MIR}^{a, b}\end{array}$ & $\begin{array}{c}\text { Ref. } \\
\text { R }\end{array}$ \\
\hline IRAS 20343+4129 IRS1 & 1.4 & $1000-3200$ & 0.8 & 0.04 & $1.3 \times 10^{-4}$ & 4.2 & $\mathrm{Y}$ & 1,2 \\
OMC1-S 137-408 & 0.50 & & 2 & 0.07 & $1.2 \times 10^{-2}$ & $0.11-3$ & $\mathrm{~N}$ & $3,4,5$ \\
L1206 & 0.91 & 1200 & 14.2 & 0.09 & $7.7 \times 10^{-5}$ & $8.0-20$ & $\mathrm{Y}$ & 6,7 \\
Cepheus E & 0.73 & 70 & 13.6 & 0.13 & $2.3 \times 10^{-4}$ & $4.0-8.0$ & $\mathrm{Y}$ & $8,9,10$ \\
IRAS 21391+5802 & 0.75 & $235-500$ & 5.1 & 0.14 & $1.4 \times 10^{-3}$ & $2.0-3.5$ & $\mathrm{Y}$ & $11,12,13,14,15$ \\
IRAS 20050+2720 A & 0.70 & 280 & 6.5 & 0.20 & $5.3 \times 10^{-4}$ & $6.5-7.3$ & $?$ & $16,17,18$ \\
OMC-2/3 MM7 & 0.45 & 76 & $0.36-0.72$ & 0.34 & $1.1 \times 10^{-3}$ & $6.3-21$ & $\mathrm{Y}$ & $19,20,21$ \\
IRAS 20293+3952 A & 2.0 & $1050^{d}$ & 4 & 2.0 & $2.1 \times 10^{-2}$ & 4.3 & $\mathrm{~N}$ & $1,22,23$ \\
IRAS 22171+5549 & 2.4 & 1800 & 25 & 2.7 & $1.2 \times 10^{-2}$ & 7 & $\mathrm{~N}$ & 24,25 \\
IRAS 21307+5049 & 3.6 & 4000 & 53 & 3.2 & $2.9 \times 10^{-3}$ & 13 & $\mathrm{~N}^{e}$ & 24,25 \\
S235 NNW-SSE & 1.8 & & & 4.0 & $1.0 \times 10^{-3}$ & $15-20$ & $\mathrm{~N}$ & 26,27 \\
S235 NE-SW & 1.8 & 1000 & 16 & 9.0 & $1.4 \times 10^{-2}$ & $5.4-14.7$ & $?$ & 26,27 \\
IRAS 23385+6053 & 4.9 & 1500 & 61 & 10.6 & $9.0 \times 10^{-3}$ & 7.3 & $\mathrm{~N}$ & 28,29 \\
HH 288 & 2.0 & 500 & $6-30$ & 11 & $1.4 \times 10^{-2}$ & 28 & $\mathrm{~N}$ & 30 \\
\hline
\end{tabular}

References: 1: Sridharan et al. (2002); 2: Palau et al. (2007b); 3: Genzel \& Stutzki (1989); 4: Zapata et al. (2006); 5: L. A. Zapata (private communication); 6: Crampton \& Fisher (1974); 7: Paper II; 8: Lefloch et al. (1996); 9: Moro-Martín et al. (2001); 10: Noriega-Crespo et al. (2004); 11: Matthews (1979); 12: Saraceno et al. (1996); 13: de Gregorio-Monsalvo (2006); 14: Paper I; 15: Getman et al. (2007); 16: Dame \& Thaddeus (1985); 17: Froebrich (2005); 18: this work; 19: Takahashi et al. (2006); 20: Takahashi et al. (2007); 21: Nielbock et al. (2003); 22: Beuther et al. (2004a); 23: Palau et al. (2007a); 24: Molinari et al. (2002); 25: Fontani et al. (2004a); 26: Evans \& Blair (1981); 27: Felli et al. (2004); 28: Molinari et al. (1998); 29: Fontani et al. (2004b); 30: Gueth et al. (2001).

${ }^{a}$ Y: source detected at $\lambda<8.0 \mu \mathrm{m}$ by the Infrared Array Camera (IRAC) of the Spitzer Space Telescope, ISOCAM at $7 \mu \mathrm{m}$, or by $2 \mathrm{MASS}$; N: source not detected at $\lambda<8.0 \mu \mathrm{m}$; ?: source for which the association between the millimeter and mid-infrared emission is dubious.

${ }^{b}$ The information was obtained either from the literature of from the Spitzer Science Center Data Archive interface Leopard.

${ }^{c}$ Parameters correspond to the whole emission, including the two outflows associated with OMC1-S 137-408 and with HH 288.

${ }^{d}$ The luminosity of the intermediate-mass YSO powering the outflow A (Palau et al. 2007a).

${ }^{e}$ The MIR emission peak at $7 \mu \mathrm{m}$ is offset by $\sim 6^{\prime \prime}$ from the millimeter and submillimeter emission and likely due to the nearby cluster (Fontani et al. 2004b).

${ }^{f}$ The MIR emission is offset by 1". 5 from the millimeter peak and it cannot be firmly established whether both infrared and millimeter sources are related (Felli et al. 2006).

yet detected at shorter wavelengths. In many cases, they are also associated with young clusters of more evolved NIR sources (e.g. IRAS 21391+5802: Nisini et al. 2001; Getman et al. 2007; L1206: Ressler \& Shure 1991; Kumar et al. 2006; IRAS 20050+2720: Chen et a. 1997; Gutermuth et al. 2005; Kumar et al. 2006; S235: Felli et al. 1997; IRAS 22172+5549: Fontani et al. 2004a; Kumar et al. 2006; IRAS 21307+5049: Fontani et al. 2004a; IRAS 20293+3952 A: Palau et al. 2007a). Interestingly, in some cases these NIR clusters contain a large number of low-mass evolved, Class II and Class III sources (e.g. IRAS 21391+5802: Getman et al. 2007, IRAS 20050+2720: Chen et al. 1997; IRAS 20293+3952 A: Palau et al. 2007a; and possibly L1206: M. S. N. Kumar, private communication). This means that the intermediate-mass protostars and these more evolved low-mass sources cannot be coeval. All this suggests that, at least in some regions, intermediate-mass protostars would start forming after the first generation of low-mass stars has completed its main accretion phase. Such a situation appears to be typical of high-mass star-forming regions, where embedded massive stars that are still accreting material are surrounded by clusters of less embedded and more evolved low-mass Class I or Class II sources (Kumar et al. 2006).

The presence of multiple sources in intermediate-mass starforming regions also explains the complex appearance of the molecular outflows in these regions when observed at low angular resolution. This is due to, first of all, the presence usually of more than one outflow in the region (e.g. HH 288: Gueth et al. 2001; IRAS 21391+5802: Paper I; IRAS 20050+2720: this paper), and second, to the stronger interaction of the outflow with the high-density clumps surrounding the intermediate-mass protostar (IRAS 21391+5802: Paper I). However, as shown by these studies of intermediate-mass starforming regions, the molecular outflows, which are intrinsically more energetic than those driven by low-mass stars, are collimated and less complex when observed at high angular resolution. In fact, they appear collimated even at low outflow velocities (Papers I and II), similar to the low-mass protostellar flow in HH 211 (Gueth \& Guilloteau 1999; Palau et al. 2006b).

\subsection{Relationships between protostar properties and outflow parameters}

Table 5 shows the properties of intermediate-mass outflows and their powering sources derived from interferometric observations, which have been compiled from the literature. Table 5 also gives information on whether the sources have been detected or not at NIR or mid-infrared (MIR) wavelengths. As seen in the table, only a very limited number of intermediate-mass outflows have been studied at high angular resolution. In spite of the poor statistics of the sample, we compared the properties of intermediate-mass YSOs with those of low-mass protostars. To do this, we checked whether intermediate-mass YSOs are consistent with the correlations between source and outflow properties found for a sample of Class 0 and Class I protostars by Bontemps et al. (1996). In particular, we checked the correlation between circumstellar envelope mass $M_{\text {env }}$ and the momentum rate in the outflow $F_{\text {out }}$, the correlation between bolometric luminosity $L_{\text {bol }}$ and $F_{\text {out }}$, and the one between the normalized momentum $F_{\text {out }} c / L_{\text {bol }}$ and $M_{\text {env }} / L_{\text {bol }}^{0.6}$ (see Fig. 8). Following Bontemps et al. (1996), we used the observed momentum rate 

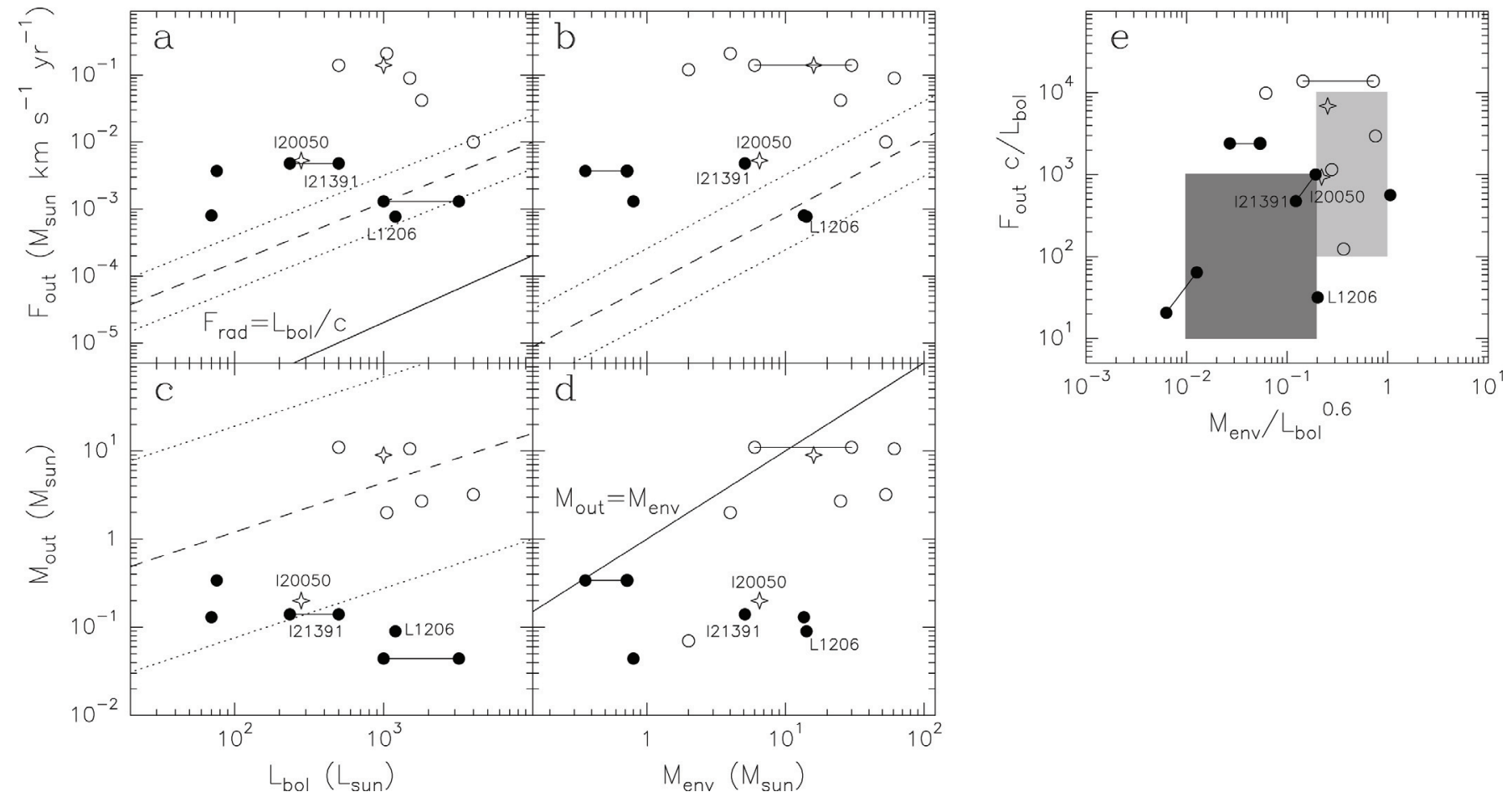

Fig. 8. Correlations between source and outflow properties for the sample of intermediate-mass protostars and outflows listed in Table 5: a) Outflow momentum flux $F_{\text {out }}$ versus bolometric luminosity $L_{\text {bol }}$. The best fit $F_{\text {out }}-L_{\text {bol }}$ correlation found for Class I sources by Bontemps et al. (1996) is plotted as a dashed line. Dotted lines represent the dispersion of the fit. The solid line presents the relation $F_{\text {out }}=L_{\text {bol }} / c$. b) $F_{\text {out }}$ versus circumstellar envelope mass $M_{\text {env }}$. The best fit $F_{\text {out }}-M_{\text {env }}$ correlation found for a low-mass Class 0 and Class I sample by Bontemps et al. (1996) is plotted as a dashed line. Dotted lines represent the dispersion of the fit. c) Outflow mass $M_{\text {out }}$ versus $L_{\text {bol }}$. The least-square linear fit found for a sample of low- to high-mass sources by Wu et al. (2004) is plotted as a dashed line. Dotted lines represent the dispersion of the fit. d) $M_{\text {out }}$ versus $M_{\text {env }}$. The solid line presents the relation $M_{\text {out }}=M_{\text {env }}$. e) $F_{\text {out }} c / L_{\text {bol }}$ (dimensionless) versus $M_{\text {env }} / L_{\text {bol }}^{0.6}\left(M_{\text {env }}\right.$ and $L_{\mathrm{bol}}$ in units of $M_{\odot}$ and $\left.L_{\odot}\right)$. The light and dark grey boxes roughly denote the zones where the Class 0 and Class I sources, respectively, are located in Fig. 7 of Bontemps et al. (1996). The horizontal and oblique lines that join two dots indicate two different values for the same source or outflow property (see Table 5). Open circles represent sources not detected at $\lambda<8 \mu \mathrm{m}$, filled circles represent sources detected at $\lambda<8 \mu \mathrm{m}$, and stars represent sources for which the association between the millimeter and mid-infrared emission is dubious (see Table. 5). The three sources studied by us in Paper I, Paper II, and this work are labeled.

in the outflow $F_{\text {obs }}$ (see Table 5), corrected for inclination and opacity. We applied a mean correction factor of 2.9 for the inclination, corresponding to a mean inclination angle with respect to the plane of the sky of $\sim 32.7^{\circ}$, and a mean correction factor of 3.5 for the opacity. Therefore, the corrected momentum flux of the outflow $F_{\text {out }}$ is $F_{\text {out }} \sim 2.9 \times 3.5 \times F_{\text {obs }} \sim 10 \times F_{\text {obs }}$. For the three molecular outflows that had already been corrected for inclination angle $i$, we only applied the opacity mean correction factor 3.5. In addition, we also compared $M_{\mathrm{env}}$ and the mass of the outflow $M_{\text {out }}$, and $L_{\text {bol }}$ and $M_{\text {out }}$. The different symbols in Fig. 8 indicate whether the sources have been detected at $\lambda<8 \mu \mathrm{m}$ or not or whether the detection is dubious (see Table 5).

In Figs. $8 \mathrm{a}$ and $8 \mathrm{~b}$, we compare the relationships between $F_{\text {out }}$ and $L_{\text {bol }}$ or $M_{\text {env }}$ obtained for the intermediate-mass outflows with those obtained by Bontemps et al. (1996). As can be seen in the plot, intermediate-mass YSOs have, in general, higher $F_{\text {out }}$ than low-mass objects. One could argue that this is due to the sources in our sample having been observed with interferometers and those of Bontemps et al. with single-dish. However, for those sources in our sample that have been observed with single-dish as well, the derived values of $F_{\text {out }}$ are either comparable to or even higher than those derived with interferometers. According to Eq. (5) of Bontemps et al. (1996), $F_{\text {out }}$ is related to the accretion rate $\dot{M}_{\text {acc }}$, the entrainment efficiency, and the outflow driving engine efficiency. Therefore, the higher
$F_{\text {out }}$ values suggest either a higher $\dot{M}_{\text {acc }}$ for intermediate-mass stars, a higher entrainment efficiency, or a higher outflow driving engine efficiency. Calvet et al. (2004) have found by means of optical and ultraviolet observations that the average mass accretion rate for a sample of intermediate-mass T Tauri stars, which are the evolutionary predecessors of Herbig Ae stars, is a factor $\sim 5$ higher than for their low-mass counterparts. Therefore, it is very likely that intermediate-mass YSOs accrete material faster. This seems a reasonable conjecture, because these protostars have to gain enough mass before the circumstellar disks are dispersed. According to Fuente et al. (2001), the dispersal of the circumstellar material and the end of the outflow activity should occur in $<10^{5}$ yrs.

As seen in Table 5 and Fig. 8, half of the sources have not been detected at $\lambda<8.0 \mu \mathrm{m}$. This suggests that these sources could be more deeply embedded in the core and, therefore, be in an earlier evolutionary stage than those detected and represented by filled circles. The difference in detection rate at NIR or MIR wavelengths is also evident in some source and outflow properties. Figure 8 shows that for a similar $L_{\mathrm{bol}}$ or $M_{\mathrm{env}}$, the sources not detected at NIR or MIR wavelengths have higher, in some cases up to $\sim 2$ orders of magnitude higher, $F_{\text {out }}$ and $M_{\text {out }}$ than those detected. Therefore, this suggests that the sources not detected at NIR or MIR wavelengths are more efficient at driving their outflows, which are more powerful, than those detected. The same happens when comparing $M_{\text {out }}$ for objects with similar 
$L_{\text {bol }}$ (see Fig. 8c). Those not detected at NIR or MIR wavelengths put more mass in the outflows than those detected. In addition, the non-detected sources agree better with the correlation between $M_{\text {out }}$ and $L_{\text {bol }}$ obtained by Wu et al. (2004) for a sample of low- to high-mass YSOs. Although the radiation pressure of the central source would not be enough to drive the outflow if the photons emitted by the central source were scattered only once, as shown by the fact that all the outflows have $F_{\text {out }}$ above the radiative momentum flux $F_{\text {rad }}=L_{\text {bol }} / c$ (see Fig. 8a), $L_{\text {bol }}$ of the central source, and $M_{\text {out }}$ are still correlated, showing the dependence of the outflow on its driving source. As seen in Fig. 8d, in general, the sources not detected at NIR or MIR wavelengths put almost the same amount of mass in the outflow as they have in the circumstellar envelope.

To further investigate these differences, we removed any luminosity dependence from both $F_{\text {out }}$ and $M_{\text {env }}$. Following Bontemps et al. (1996), we plotted the outflow efficiency $F_{\text {out }} c / L_{\text {bol }}$ versus $M_{\text {env }} / L_{\text {bol }}^{0.6}$ (Fig. $8 \mathrm{e}$ ), along with the zones where the low-mass Class 0 and Class I sources are roughly located in Fig. 7 of Bontemps et al. (1996). This plot shows that, in general, the sources not detected at NIR or MIR wavelengths have higher outflow efficiency. In the low-mass regime, there is a decline in the outflow efficiency with evolutionary stage (Bontemps et al. 1996). Therefore, although we do not claim that the sources not detected at $\lambda<8.0 \mu \mathrm{m}$ are like low-mass Class 0 objects, we propose that these sources could be younger than those detected, as additionally suggested by the differences in properties such as $F_{\text {out }}, M_{\text {out }}$, and outflow efficiency. In the low-mass regime, the youngest protostars have been classified as Class 0 objects, based on criteria such as the ratio of submillimeter to bolometric luminosity, $L_{\text {submm }} / L_{\text {bol }}>5 \times 10^{-3}$, where $L_{\text {submm }}$ is measured longwards of $350 \mu \mathrm{m}$ (André et al. 1993). However, intermediate-mass star-forming regions are located, in general, at farther distances than low-mass ones and usually associated with small clusters. Hence, the luminosity at wavelengths longwards of $350 \mu \mathrm{m}$ will likely have contributions from other sources in the cluster, and therefore the criterion used to classify the youngest low-mass sources might not be applicable for intermediate-mass ones. In order to confirm this possible evolutionary difference in the sources of our sample, one should conduct a detailed source-by-source study of the SED at high angular resolution; however, this goes beyond the scope of this work.

\section{Conclusions}

We studied the dust at 2.7 and $7 \mathrm{~mm}$ and the gas at $2.7 \mathrm{~mm}$ towards IRAS 20050+2720, an intermediate-mass YSO, with the OVRO Millimeter Array and the VLA. We also attempted to put the results on this and previously studied intermediate-mass sources in the context of intermediate-mass star formation.

The $2.7 \mathrm{~mm}$ continuum emission was resolved into three sources, OVRO 1, OVRO 2, and OVRO 3. Two of them, OVRO 1 and OVRO 2, were also detected at $7 \mathrm{~mm}$. OVRO 3, which is located close to the $\mathrm{C}^{18} \mathrm{O}$ emission peak, could be associated with IRAS $20050+2720$. The strongest source at millimeter wavelengths is OVRO 1 , which has a deconvolved diameter of $\sim 3^{\prime \prime} .3$ ( $\sim 2300 \mathrm{AU})$ at the half-peak intensity contour of the dust emission. The source is surrounded by an extended and a fairly spherical halo or envelope, which has a size of $\sim 11^{\prime \prime} .3$ ( $~ 8000$ AU) at the $3 \sigma$ contour level. OVRO 2 has a deconvolved diameter of $\sim 2$.'3 ( 1600 AU) at the half-peak intensity contour of the dust emission, and OVRO 3 is not resolved. The mass of the sources, estimated from the dust continuum emission, is $6.5 M_{\odot}$ for OVRO $1,1.8 M_{\odot}$ for OVRO 2 , and $1.3 M_{\odot}$ for OVRO 3 .

The multiplicity of sources observed towards IRAS 20050+2720, as well as towards IRAS $21391+5802$ and L1206, appears to be typical of intermediate-mass star-forming regions. Intermediate-mass stars form in dense clustered environments, and they are associated in most cases with small protoclusters of low-mass millimeter sources, some of them not yet detected at shorter wavelengths. In many cases, as for example IRAS 20050+2720, IRAS 21391+5802, and L1206, intermediate-mass embedded protostars are also associated with young clusters of more evolved sources with detectable NIR emission. This NIR clusters sometimes contain a large number of low-mass evolved, Class II and Class III sources. This suggests that, at least in some regions, intermediate-mass protostars would start forming after the first generation of low-mass stars has completed its main accretion phase, a situation that appears to be typical of high-mass star-forming regions (Kumar et al. 2006).

The CO $(J=1 \rightarrow 0)$ emission towards IRAS $20050+2720$ traces two bipolar outflows within the OVRO field of view, a roughly east-west bipolar outflow, coincident with the outflow A of Bachiller et al. (1995) and apparently driven by the intermediate-mass source OVRO 1, and a northeast-southwest bipolar outflow, labeled outflow B and probably powered by a YSO engulfed in the circumstellar envelope surrounding OVRO 1. The outflow A presents a different morphology for the blueshifted and the redshifted emission. The blueshifted emission is more compact than the redshifted one. This lack of symmetry between the lobes could be due to the presence of the OVRO 3 core.

High-angular resolution observations show that, in general, intermediate-mass outflows, which are intrinsically more energetic than those driven by low-mass YSOs, are not intrinsically more complex than low-mass outflows. Intermediate-mass outflows appear collimated, even at low velocities, and have properties that do not differ significantly from those of lowmass stars. The momentum rate in the outflow $F_{\text {out }}$ values of intermediate-mass protostars are higher than those of low-mass ones. Although one cannot discard a higher entrainment efficiency or outflow-driving engine efficiency, this suggests that intermediate-mass YSOs very likely accrete material faster than low-mass ones.

Known intermediate-mass protostars do not form a homogeneous group but have different properties. The sources not been detected at $\lambda<8.0 \mu \mathrm{m}$ clearly have higher $F_{\text {out }}$, mass of the outflow $M_{\text {out }}$, and outflow efficiency than the sources detected at $\lambda<8.0 \mu \mathrm{m}$ for similar $M_{\mathrm{env}}$ and $L_{\mathrm{bol}}$. This suggests that the sources not detected at NIR or MIR wavelengths, which are likely to be more embedded in the core and to drive more powerful outflows, could be in an earlier evolutionary stage.

Acknowledgements. It is a pleasure to thank the OVRO staff for their support during the observations. MTB is deeply grateful to Francesco Fontani, Fabrizio Massi, Aina Palau, M. S. Nanda Kumar, Luis Zapata, and Satoko Takahashi for the information provided on intermediate-mass protostars. MTB, RE, JMG, and GA are supported by MEC grant AYA2005-08523-C03. JMG acknowledges support from AGAUR grant SGR 00489. GA acknowledges support from Junta de Andalucía. This research has made use of the NASA/IPAC Infrared Science Archive, which is operated by the Jet Propulsion Laboratory, California Institute of Technology, under contract with the National Aeronautics and Space Administration (NASA). This publication makes use of data products from the Two Micron All Sky Survey, which is a joint project of the University of Massachusetts and the IPAC/California Institute of Technology, funded by NASA and the National Science Foundation. 


\section{References}

André, P., Ward-Thompson, D., \& Barsony, M. 1993, ApJ, 406, 122

Anglada, G., Rodríguez, L. F., \& Torrelles, J. M. 1998, in Star Formation with the Infrared Space Observatory, ed. J. L. Yun \& R. Liseau (San Francisco: ASP), ASP Conf. Ser., 132, 303

Arce, H. G., \& Goodman, A. A. 2001, ApJ, 554, 132

Bachiller, R. 1996, ARA\&A, 34, 111

Bachiller, R., Fuente, A., \& Tafalla, M. 1995, ApJ, 445, L51

Beltrán, M. T., Girart, J. M., Estalella, R., Ho, P. T. P., \& Palau, A. 2002, ApJ, 573, 246 (Paper I)

Beltrán, M. T., Girart, J. M., Estalella, R., \& Ho, P. T. P. 2004a, A\&A, 426, 941

Beltrán, M. T., Gueth, F., Guilloteau, S., \& Dutrey, A. 2004b, A\&A, 416, 631

Beltrán, M. T., Brand, J., Cesaroni, R., et al. 2006a, A\&A, 447, 221

Beltrán, M. T., Girart, J. M., \& Estalella, R. 2006b, A\&A, 457, 865 (Paper II)

Beuther, H., Schilke, P., \& Gueth, F. 2004, ApJ, 608, 330

Bontemps, S., André, P., Terebey, S, \& Cabrit, S. 1996, A\&A, 311, 858

Briggs, D. 1995, Ph.D. Thesis, New Mexico Inst. Mining \& Tech.

Calvet, N., Muzerolle, J., Briceño, C., et al. 2004, ApJ, 128, 1294

Chen, H., Tafalla, M., Greene, T. P., Myers, P. C., \& Wilner, D. J. 1997, ApJ, 475,163

Chini, R., Ward-Thompson, D., Kirk, J. M., et al. 2001, A\&A, 369, 155

Choi, M., Panis, J.-F., \& Evans II, N. J., 1999, A\&AS, 122, 519

Crampton, D., \& Fisher, W. A. 1974, Publ. Dominion Ap. Obs. Victoria, 14

Dame, T. M., \& Thaddeus, P. 1985, ApJ, 297, 751

de Gregorio-Monsalvo, I., Gómez, J. F., Suárez, O., et al. 2006, ApJ, 642, 319

de Vicente, P., Martín-Pintado, J., Neri, R., \& Colom, P. 2000, A\&A, 361, 1058

Evans, N. J. II, \& Blair, G. N. 1981, ApJ, 246, 394

Frerking, M. A., Langer, W. D., \& Wilson, R. W. 1982, ApJ, 262, 590

Felli, M., Testi, L., Valdettaro, R., \& Wang, J.-J. 1997, A\&A, 320, 5942002 , A\&A, 420, 553

Felli, M., Massi, F., Navarrini, A., et al. 2004, A\&A, 420, 553

Felli, M., Massi, F., Robberto, M., \& Cesaroni, R. 2006, A\&A, 453, 911

Fontani, F., Cesaroni, R., Testi, L., et al. 2004a, A\&A, 424, 179

Fontani, F., Cesaroni, R., Testi, L., et al. 2004b, A\&A, 414, 299

Froebrich, D. 2005, ApJS, 156, 169

Fuente, A., Neri, R., Martín-Pintado, J., et al. 2001, A\&A, 366, 873

Fuente, A., Ceccarelli, C., Neri, R., et al. 2007, A\&A, 468, L37

Furuya, R. S., Kitamura, Y., Wootten, A., Claussen, M. J., Kawabe, R. 2003, ApJS, 144, 71

Furuya, R. S., Kitamura, Y., Wootten, A., Claussen, M. J., \& Kawabe, R. 2005, A\&A, 438, 571

Genzel, R., \& Stutzki, J. 1989, ARA\&A, 27, 41

Getman, K., Feigelson, E. D., Garmire, G., Broos, P., \& Wang, J. 2007, ApJ, 654,316

Gueth, F., \& Guilloteau, S. 1999, A\&A, 343, 571

Gregersen, E. M., Evans, N. J. II, Zhou, S., \& Choi, M. 1997, ApJ, 484, 256

Gueth, F., Schilke, P., \& McCaughrean, M. J. 2001, A\&A, 375, 1018

Gutermuth, R. A., Megeath, T., Pipher, J. L., et al. 2005, ApJ, 632, 397

Hildebrand, R. H. 1983, QJRAS, 24, 267

Hogerheijde, M. R., van Dishoeck, E. F., Blake, G. A., \& van Langevelde, H. J. 1997, ApJ, 489, 293

Hogerheijde, M. R., van Dishoeck, E. F., Salverda, J. M., \& Blake, G. A. 1999, ApJ, 513, 350

Kulesa, C. A., Hungerford, A. L., Walker, C. K., Zhang, X., \& Lane, A. P. 2005 , ApJ, 625, 194
Kumar, M. S. N., Keto, E., \& Clerkin, E. 2006, A\&A, 449, 1033

Laundhart, R., \& Henning, Th. 1997, A\&A, 326, 329

Lefloch, B., Eislöffel, J., \& Lazareff, B. 1996, A\&A, 313, L17

Looney, L. W., Mundy, L. G., \& Welch, W. J. 2000, ApJ, 529, 477

MacLeod, G. C., Scalise, E. Jr., Saedt, S., Galt, J. A., \& Gaylard, M. J. 1998, AJ, 116,1897

Martín-Pintado, J., Jiménez-Serra, I., Rodríguez-Franco, A., Martín, S., \& Thum, C. 2005, ApJ, 628, L61

Matthews, T. J. 1979, A\&A, 75, 345

Molinari, S., Brand, J., Cesaroni, R., \& Palla, F. 1996, A\&A, 308, 573

Molinari, S., Testi, L., Brand, J., Cesaroni, R., \& Palla, F. 1998, ApJ, 505, L39

Molinari, S., Testi, L., Rodríguez, L. F., \& Zhang, Q. 2002, ApJ, 570, 758

Moro-Martín, A., Noriega-Crespo, A., Molinari, S., et al. 2001, ApJ, 555, 146

Neri, R., Fuente, A., Ceccarelli, C., et al. 2007, A\&A, 468, L33

Nielbock, M., Chini, R., \& Müller, S. A. H. 2003, A\&A, 408, 245

Nisini, B., Massi, F., Vitali, et al. 2001, A\&A, 376, 553

Noriega-Crespo, A., Moro-Martín, A., Carey, S., et al. 2004, ApJSS, 154, 402

Ossenkopf, V., \& Henning, Th. 1994, A\&A, 291, 943

Palau, A. 2006, Ph.D. Thesis, Universitat de Barcelona

Palau, A., Estalella, R., Ho, P. T. P., \& Estalella, R. 2006, ApJ, 636, L137

Palau, A., Estalella, R., Girart, J. M., et al. 2007a, A\&A, 465, 219

Palau, A., Ho, P. T. P., Zhang, Q., Beuther, H., \& Beltrán, M. T. 2007b, A\&A, 474, 911

Palla, F., Brand, J., Cesaroni, R., Comoretto, G., \& Felli, M. 1991, A\&A, 246, 249

Ressler, M. E., \& Shure, M. 1991, AJ, 102, 1398

Richer, J., Shepherd, D. S., Cabrit, S., Bachiller, R., \& Churchwell, E. 2000, in Protostars and Planets IV, ed. V. Mannings, A. Boss, \& S. Russell (Tucson: University of Arizona Press), 867

Ridge, N. A., Wilson, T. L., Megeath, S. T., Allen, L. E., \& Myers, P. C. 2003, AJ, 126, 286

Saraceno, P., Ceccarelli, C., Clegg, P., et al. 1996, A\&A, 315, L293

Scoville, N. Z., Carlstrom, J. E., Chandler, C. J., et al. 1993, PASP, 105, 1482

Scoville, N. Z., Sargent, A. I., Sanders, D. B., et al. 1986, ApJ, 303, 416

Shepherd, D. S. 2005, in Massive Star Birth: A Crossroads of Astrophysics, ed. R. Cesaroni, M. Felli, E. Churchwell, \& M. Walmsley (Cambridge: Cambridge University Press), Proc. IAU Symp., 227, 237

Shepherd, D. S., \& Churchwell, E. 1996a, 457, 267

Shepherd, D. S., \& Churchwell, E. 1996b, 472, 225

Schneider, N., Bontemps, S., Simon, R., et al. 2006, A\&A, 458, 855

Sridharan, T. K., Beuther, H., Schilke, P., Menten, K. M.., \& Wyrowski, F. 2002, ApJ, 566, 931

Takahashi, S., Saito, M., Takakuwa, S., \& Kawabe, R. 2006, ApJ, 651, 933

Takahashin, S., et al. 2007, in preparation

Vanden Bout, P. A., Loren, R. B., Snell, R. L., \& Wootten, A. 1983, ApJ, 271, 161

Wilking, B. A., Mundy, L. G., Blackwell, J. H., \& Howe, J. E. 1989, ApJ, 345, 257

Williams, J. P., \& Myers, P. C. 1999, ApJ, 511, 208

Wu, Y., Wei, Y., Zhao, M., et al. 2004, A\&A, 426, 503

Zapata, L. A., Ho, P. T. P., Rodríguez, L. F., et al. 2006, ApJ, 653, 398

Zhang, Q., Hunter, T. R., Brand, J., et al. 2001, ApJ, 552, 167

Zhang, Q., Hunter, T. R., Brand, J., et al. 2005, ApJ, 625, 864 